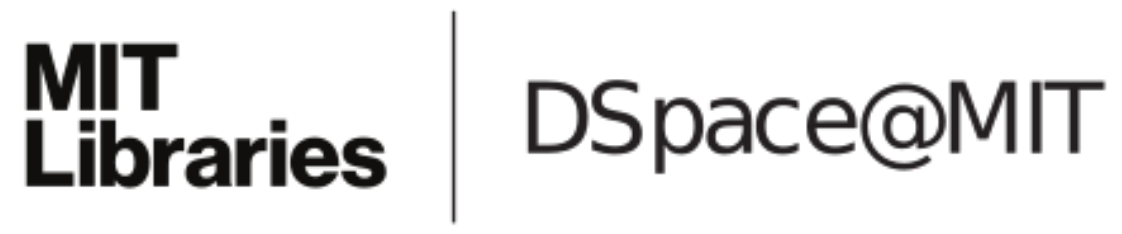

\author{
MIT Open Access Articles
}

Competing Engines of Growth: Innovation and Standardization

The MIT Faculty has made this article openly available. Please share how this access benefits you. Your story matters.

Citation: Acemoglu, Daron, Gino Gancia, and Fabrizio Zilibotti. "Competing engines of growth: Innovation and standardization\#." Journal of Economic Theory (2010): n. pag. Copyright (c 2010 Published by Elsevier Inc.

As Published: http://dx.doi.org/10.1016/j.jet.2010.09.001

Publisher: Elsevier ScienceDirect

Persistent URL: http://hdl.handle.net/1721.1/61359

Version: Author's final manuscript: final author's manuscript post peer review, without publisher's formatting or copy editing

Terms of use: Creative Commons Attribution-Noncommercial-Share Alike 3.0 


\title{
Competing Engines of Growth: Innovation and Standardization*
}

\author{
Daron Acemoglu, ${ }^{\dagger}$ Gino Gancia, ${ }^{\ddagger}$ and Fabrizio Zilibotti ${ }^{\S}$
}

April 2010, Revised August 2010.

\begin{abstract}
We study a dynamic general equilibrium model where innovation takes the form of the introduction of new goods whose production requires skilled workers. Innovation is followed by a costly process of standardization, whereby these new goods are adapted to be produced using unskilled labor. Our framework highlights a number of novel results. First, standardization is both an engine of growth and a potential barrier to it. As a result, growth is an inverse U-shaped function of the standardization rate (and of competition). Second, we characterize the growth and welfare maximizing speed of standardization. We show how optimal protection of intellectual property rights affecting the cost of standardization vary with the skill-endowment, the elasticity of substitution between goods and other parameters. Third, we show that, depending on how competition between innovating and standardizing firms is modelled and on parameter values, a new type of multiplicity of equilibria may arise. Finally, we study the implications of our model for the skill-premium and we illustrate novel reasons for linking North-South trade to intellectual property rights protection.
\end{abstract}

JEL classification: J24, O31, O33, O34, O41.

Keywords: competition policy, growth, intellectual property rights, skilled labor, standardization, technology adoption,.

${ }^{*}$ We thank seminar participants at the SED Annual Meeting (Boston, 2008), Bank of Italy, the Kiel Institute, CERGE-EI, Univesity of Alicante and the REDg-Dynamic General Equilibrium Macroeconomics Workshop (Madrid, 2008) for comments. We are also grateful to Karl Shell and two anonymous referees for useful suggestions. Gino Gancia acknowledges financial support from the Barcelona GSE, the Government of Catalonia and the ERC Grant GOPG 240989. Fabrizio Zilibotti acknowledges financial support from the ERC Advanced Grant IPCDP-229883.

$\dagger^{\dagger} \mathrm{MIT}$ and Canadian Institute for Advanced Research. Email: daron@mit.edu.

${ }^{\ddagger}$ CREI and CEPR. Email: ggancia@crei.cat.

$\S$ University of Zurich and CEPR. Email: zilibott@iew.uzh.ch. 


\section{InTRODUCTION}

The diffusion of new technologies is often coupled with standardization of product and process innovations. New technologies, when first conceived and implemented, are often complex and require skilled personnel to operate. At this stage, their use in the economy is limited both by the patents of the innovator and the skills that these technologies require. Their widespread adoption and use necessitates the tasks involved in these new technologies to become more routine and standardized, ultimately enabling their cheaper production using lower-cost unskilled labor. However, such standardization not only expands output but also implies that the rents accruing to innovators will come to an end. Therefore, the process of standardization is both an engine of economic growth and a potential discouragement to innovation. In this paper, we study this interplay between innovation and standardization.

The history of computing illustrates the salient patterns of this interplay. The use of silicon chips combined with binary operations were the big breakthroughs, starting the information communication technology (ICT) revolution. During the first 30 years of their existence, computers could only be used and produced by highly skilled workers. Only a lengthy process of standardization made computers and silicon chips more widely available and more systematically integrated into the production processes, to such a degree that today computers and computer-assisted technologies are used at every stage of production with workers of very different skill levels. At the same time that the simplification of manufacturing processes allowed mass production of electronic devices and low prices, competition among ICT firms intensified enormously, first among few industry leaders and then more broadly at a global scale.

More generally, the business literature has documented a common pattern in the life-cycle of industries. New industries are often highly concentrated due to the complexity of their products. Over time, both entry and process innovation intensify until the introduction, often by newcomers, of a "dominant standard" facilitates more standardized, large-scale production and erodes the monopoly advantage of incumbent firms. For instance, in the early 1960s the American calculator industry consisted of five major companies producing complex and expensive electronic machines with more than 2300 parts. These companies lost most of their market share after the introduction, in 1971, of the calculator on a chip, which made the assembly of units extremely simple - merely piecing together the chip, display device and keyboard (Utterback and Suarez, 1993). Similarly, although the production of transistors was initiated in 1947 by Bell Laboratories, the first industry standard, the planar transistor, was introduced in 1959 by the newly founded company Fairchild Semiconductor. The great advantage of this design was its flat surface, on which electrical connections could be achieved by depositing an evaporated metal film; previously this process required skill manual work on the irreg-

ular surface of the mesa transistor (Utterback and Suarez, 1993). Another example is provided by the introduction of the Banbury Mixer in the tire industry, which elimi- 
nated the slow and hazardous process of mixing rubber with other compounds, paving the way for mass production and forcing many incumbent firms to exit (Jovanovic and MacDonald, 1994).

In our model, new products are invented via costly R\&D and can first be produced only by skilled workers. This innovation process is followed by a costly process of standardization, whereby the previously new goods are adapted to be produced using unskilled labor. ${ }^{1}$ Free entry into standardization makes it a competing process; due to the familiar Arrow effect, standardization will be undertaken by newcomers, which may then displace incumbent producers. By shifting some technologies to low-skill workers, standardization alleviates the pressure on scarce high-skill workers, thereby raising aggregate demand and fostering incentives for further innovation. Yet, the anticipation of standardization also reduces the potential profits from new products, discouraging innovation. This implies that while standardization - and the technology adoption that it brings - is an engine of economic growth, it can also act as a barrier to growth by potentially slowing down innovation.

Our baseline framework provides a simple model for the analysis of this interplay. Under some relatively mild assumptions, we establish the existence of a unique balanced growth path equilibrium that is saddle-path stable. We show that equilibrium growth is an inverse U-shaped function of the "extent of competition" captured by the cost of standardization. When standardization is very costly, growth is relatively slow because new products use skilled workers for a long while and this reduces their scale of production and profitability. On the other hand, when standardization is very cheap, growth is again relatively slow, this time because innovators enjoy ex post profits only for a short while. This inverse U-shaped relationship between competition and growth is consistent with the empirical findings in Aghion et al. (2005), and complements the theoretical channel highlighted in Aghion et al. (2001, 2005), which is driven by the interplay of their "escape competition" mechanism and the standard effects of monopoly profits on innovation.

In our model, the laissez-faire equilibrium is inefficient for two reasons. First, as in many models of endogenous technology, there is an appropriability problem: both innovating and standardizing firms are able to appropriate only a fraction of the gain in consumer surplus created by their investment and this makes the growth rate too low. Second, there is a new form of "business stealing" effect, whereby the costly standardization decisions reduce the rents of innovators. ${ }^{2}$ The possibility that the laissez-faire

\footnotetext{
${ }^{1}$ This view has a clear antecedent in Nelson and Phelps (1966), which we discuss further below. See also Autor, Levy and Murnane (2003) on the comparative advantage of unskilled workers in routine, or in our language "standardized," tasks. We can also interpret innovation as product innovation and standardization as process innovation. Evidence that firms engaging in product innovation (e.g., Cohen and Klepper, 1996) are smaller and more skill intensive than firms engaging in process innovation is consistent with our assumptions.

${ }^{2}$ Another form of business stealing, studied extensively in Schumpeterian models of vertical inno-
} 
equilibrium is inefficient and growth is maximized by intermediate levels of competition implies that welfare and growth maximizing policies are not necessarily those that provide maximal intellectual property rights (IPR) protection to innovators. Under the assumption that a government can affect markups and the cost of standardization by regulating IPR protection, we characterize growth and welfare maximizing combinations of IPR and competition policies. ${ }^{3}$ In contrast to most of the literature, the optimal policy is not the result of a trade-off between the static cost of monopoly power and dynamic gains. Rather, in our model an excess of property right protection may harm growth by increasing the overload on skilled workers, which are in short supply.

When the discount rate is small, we find that growth and welfare maximizing IPR policy involves lower protection when $\mathrm{R} \& \mathrm{D}$ costs (for new products) are lower, when markups for new products are higher and when the ratio of skilled to unskilled labor supply is greater. The latter comparative static result is a consequence of the fact that when there is a large supply of unskilled labor, standardization becomes more profitable and thus innovators require greater protection against standardization. We also show that when competition policy as well as IPR policy can be used, the optimal combination of policies involves no limits on monopoly pricing for new products, increased competition for standardized products, and lower IPR protection than otherwise. Intuitively, lower IPR protection minimizes wasteful entry costs, but this may lead to excessive standardization and weak incentives to innovate. To maximize growth or welfare, this latter effect needs to be counteracted by lower markups for standardized products. We also show that trade liberalization in less-developed countries may create negative effects on growth by changing the relative incentives to innovate and standardize. However, if increased trade openness is coupled with optimal IPR policy, it always increases welfare and growth.

Finally, we show that under different assumptions on competition between innovators and standardizers, a new type of multiplicity of equilibria (of balanced growth paths) arises. When too much of the resources of the economy are devoted to standardization, expected returns from innovation are lower and this limits innovative activity. Expectation of lower innovation reduces interest rates and encourages further standardization. Consequently, there exist equilibria with different levels (paths) of innovation and standardization. It is noteworthy that this multiplicity does not rely on technological complementarities (previously studied and emphasized in the literature); instead,

vation (e.g., Aghion and Howitt 1992), is when a monopoly is destroyed by new firms introducing a "better" version of an existing products. We suggest that standardization is also an important source of business stealing.

${ }^{3}$ In contrast to Loury (1979) and Romer (1990), we do not assume that patents protect monopoly rents forever. Instead, as in Schumpeterian models such as Aghion and Howitt (1992) and Grossman and Helpman (1991), we assume that monopoly power is eroded over time through imitation and further innovation (in particular, standardization). In this context, it is also natural to model IPR protection as a barrier to entry against standardization. 
it has much more of the flavor of "self-fulfilling equilibria," whereby the relative prices change in order to support equilibria consistent with initial expectations.

Our paper is related to several different literatures. In addition to the endogenous growth and innovation literatures (e.g., Aghion and Howitt, 1992, Grossman and Helpman, 1991, Romer, 1990, Segerstrom, Anant and Dinopoulos, 1990, Stokey, 1991), there are now several complementary frameworks for the analysis of technology adoption. These can be classified into three groups. The first includes models based on Nelson and Phelps's (1966) important approach, with slow diffusion of technologies across countries (and across firms), often related to the human capital of the workers employed by the technology adopting firms. This framework is incorporated into different types of endogenous growth models, for example, in Howitt (2000), Acemoglu, Aghion and Zilibotti (2006), and Acemoglu (2009, Chapter 18). Several papers provide more microeconomic foundations for slow diffusion. These include, among others, Jovanovic and Lach (1989), Chari and Hopenhayn (1991), Jovanovic and Nyarko (1996), Jovanovic (2009) and Galor and Tsiddon (1997), which model either the role of learning or human capital in the diffusion of technologies. The second group includes papers emphasizing barriers to technology adoption. Parente and Prescott (1994) is a well-known example. Acemoglu (2005) discusses the political economy foundations of why some societies may choose to erect entry barriers against technology adoption. The final group includes models in which diffusion of technology is slowed down or prevented because of the inappropriateness of technologies invented in one part of the world to other countries (see, e.g., Acemoglu and Zilibotti, 2001, Atkinson and Stiglitz, 1969, Basu and Weil, 1998 and David, 1975). Gancia and Zilibotti (2009) and Gancia, Müller and Zilibotti (2010) build and estimate a unified framework for studying technology diffusion in models of endogenous technical change. Our emphasis on standardization, rather than learning or other barriers to adoption, is different from, though complementary to, all three groups of papers.

Our paper is also related to Krugman's (1979) model of North-South trade and technology diffusion, whereby the South adopts new products with a delay. Krugman, in turn, was inspired by Vernon's (1966) model of the product cycle and his approach has been further extended by Grossman and Helpman (1991) and Helpman (1993). ${ }^{4}$ Our approach differs from all these models because innovation and standardization make different use of skilled and unskilled workers and because we focus on a closed economy general equilibrium setup rather than the interactions between technologically advanced and backward countries as in these papers. A new implication of our alternative set of assumptions is that, differently from previous models, growth is an inverse- $U$ function of standardization. More importantly, none of the above paper characterizes the optimal IPR policy and how it varies with skill abundance.

\footnotetext{
${ }^{4}$ Similar themes are also explored in Bonfiglioli and Gancia (2008), Antras (2005), Dinopoulos and Segerstrom (2007, 2009), Lai (1998), Yang and Maskus (2001).
} 
Our paper is also related to the literature on IPR policy (see, e.g., Klemperer, 1990, Gilbert and Shapiro, 1990, and Scotchmer, 1999, as well as Bhattacharya and Guriev, 2006, and Bessen and Maskin, 2009, for more recent contributions). In contrast to much of the existing literature, we focus on general equilibrium effects. Grossman and Lai (2004) and Boldrin and Levine (2005) are also related as they analyze the incentives that governments have to protect intellectual property in a trading economy, but do not study standardization.

Finally, our emphasis on the role of skilled workers in the production of new goods and unskilled workers in the production of standardized goods makes our paper also related to the literature on technological change and wage inequality; see, among others, Acemoglu (1998, 2003), Aghion, Howitt and Violante (2002), Caselli (1999), Galor and Moav (2000), Greenwood and Yorukoglu (1997), and Krusell, Ohanian, Rios-Rull and Violante (2000). The approach in Galor and Moav (2000) is particularly related, since their notion of ability-biased technological change also generates predictions for wage inequality similar to ours, though the economic mechanism and other implications are very different.

The rest of the paper is organized as follows. Section 2 builds a dynamic model of endogenous growth through innovation and standardization. It provides conditions for the existence, uniqueness and stability of a dynamic equilibrium with balanced growth and derives an inverse- $\mathrm{U}$ relationship between the competition from standardized products and growth. Section 3 presents the welfare analysis. After studying the first best allocation, it characterizes growth and welfare maximizing IPR and competition policies as functions of the parameters. As an application of these results, we discuss how trade liberalization in less developed countries affects innovation, standardization and optimal policies. Section 4 shows how a version of our baseline model with a different assumption on competition between incumbents and entrants may generate multiple equilibria and poverty traps. Section 5 concludes.

\section{A Model of Growth through Innovation and Standardization}

\subsection{PREFERENCES}

The economy is populated by infinitely-lived households who derive utility from consumption $C_{t}$ and supply labor inelastically. Households are composed by two types of agents: high-skill workers, with aggregate supply $H$, and low-skill workers, with aggregate supply $L$. The utility function of the representative household is:

$$
U=\int_{0}^{\infty} e^{-\rho t} \log C_{t} d t,
$$

where $\rho>0$ is the discount rate. The representative household sets a consumption plan to maximize utility, subject to an intertemporal budget constraint and a No-Ponzi game 
condition. The consumption plan satisfies the standard Euler equation:

$$
\frac{\dot{C}_{t}}{C_{t}}=r_{t}-\rho
$$

where $r_{t}$ is the interest rate. Time-indexes are henceforth omitted when this causes no confusion.

\subsection{Technology and Market Structure}

Aggregate output, $Y$, is a CES function defined over a measure $A$ of goods available in the economy. As in Romer (1990), the measure of goods $A$ captures the level of technological knowledge that grows endogenously through innovation. However, we assume that, upon introduction, new goods involve complex technologies that can only be operated by skilled workers. After a costly process of standardization, the production process is simplified and the good can then be produced by unskilled workers too. Despite this change in the production process, good characteristics remain unaltered so that all varieties contribute to final output symmetrically. Thus, $Y$ is defined as:

$$
Y=Z\left(\int_{0}^{A} x_{i}^{\frac{\epsilon-1}{\epsilon}} d i\right)^{\frac{\epsilon}{\epsilon-1}}=Z\left(\int_{0}^{A_{L}} x_{L, i}^{\frac{\epsilon-1}{\epsilon}} d i+\int_{0}^{A_{H}} x_{H, i}^{\frac{\epsilon-1}{\epsilon}} d i\right)^{\frac{\epsilon}{\epsilon-1}}
$$

where $A_{H}$ is the measure of hi-tech goods, $A_{L}$ is the measure of low-tech (standardized) goods and $A=A_{H}+A_{L} \cdot \epsilon>1$ is the elasticity of substitution between goods. The term $Z \equiv A^{\frac{\epsilon-2}{\epsilon-1}}$ introduces an aggregate externality that ensures the existence of a balanced growth path. In particular, this term ensures that output is linear in technology $A$. To see this, suppose that $x_{i}=X / A$, then $Y=A^{\frac{\epsilon-2}{\epsilon-1}} \times A^{\frac{1}{\epsilon-1}} \times X=A X$. When $\epsilon=2$, this externality disappears. Note that other endogenous growth models that do not feature the $Z$ term and allow for $\epsilon \neq 2$ (e.g., Grossman and Helpman, 1991) instead impose a similar externality in the R\&D technology (i.e., in the innovation possibilities frontier). Having the externality in the production good function instead of the R\&D technology is no less general and simplifies our analysis.

From (2), the relative demand for any two goods $i, j \in A$ is:

$$
\frac{p_{i}}{p_{j}}=\left(\frac{x_{i}}{x_{j}}\right)^{-1 / \epsilon}
$$

We choose $Y$ to be the numeraire, implying that the minimum cost of purchasing one unit of $Y$ must be equal to one:

$$
1=A^{-1}\left(\frac{1}{A} \int_{0}^{A}\left(p_{i}\right)^{1-\epsilon} d i\right)^{1 /(1-\epsilon)}
$$


Each hi-tech good is produced by a monopolist with a technology that requires one unit of skilled labor per unit of output. Each low-tech good is produced by a monopolist with a technology that requires one unit of labor per unit of output. Thus, the marginal cost is equal to the wage of skilled workers, $w_{H}$, for hi-tech firms and the wage of unskilled workers, $w_{L}$, for low-tech firms. Since high-skill worker can be employed by both highand low-tech firms, in equilibrium we must have $w_{H} \geq w_{L}$.

When standardization occurs, there are two potential producers (a high- and a lowtech one) for the same variety. The competition between these producers is described by a sequential entry-exit game. ${ }^{5}$ In stage (i) a low-tech firm can enter and produce a standardized version of the intermediate variety. Then in stage (ii), the incumbent decides whether to exit or fight the entrant. Exit is assumed to be irreversible, which implies that after leaving the market, a hi-tech firm cannot return and the low-tech entrant becomes a monopolist. If the incumbent does not exit, the two firms compete à la Bertand (stage (iii)). We assume that all firms entering stage (iii) must incur a "minimum cost" $\xi>0$, because they are committed to producing a small amount. Without this assumption, stage (ii) would be vacuous, as incumbents would have a "weakly dominant" strategy of staying in and producing $x=0$ in stage (iii). ${ }^{6}$ The presence of this minimum cost ensures that the dominant firm will be able to charge a monopoly (rather than limit) price, simplifying the analysis. Provided that $\xi$ is sufficiently low, the presence of the minimum cost has no other consequences for the equilibrium.

Regardless of the behavior of other producers or other prices in this economy, a subgame-perfect equilibrium of this game must have the following features: standardization in sector $j$ will be followed by the exit of the high-skill incumbent whenever $w_{H}>w_{L}$. If the incumbent did not exit, competition in stage (iii) would result in all of the market being captured by the low-tech firm due to its cost advantage and the incumbent would make a loss (as $\xi>0$ ). Thus, as long as the skill premium is positive, firms contemplating standardization can ignore any competition from incumbents. However, if $w_{H}<w_{L}$ (although this case cannot happen in equilibrium) incumbents would fight entrants and can dominate the market. Anticipating this, standardization is not profitable in this case and will not take place. Finally, in the case where $w_{H}=w_{L}$, there is a potential multiplicity of equilibria, where the incumbent is indifferent between fighting and exiting. In what follows, we will ignore this multiplicity and adopt the tie-breaking rule that in this case the incumbent fights. This tie-breaking rule ensures

\footnotetext{
${ }^{5}$ We assume that, as in Schumpeterian models of technological change, standardization does not infrige upon existing patents. Instead, we assume below that stricter IPR protection directly increases the cost of standardization (see Section 3).

${ }^{6}$ Such a cost of staying in business can be given various different interpretations. For instance, each firm that decides to stay in the market in stage (ii) may be committed to produce $\xi \equiv \Xi / A$ units of output. We could alternatively assume that each active firm may must incur a fixed cost of operations. In this case, we must assume the cost to be infinitesimal $(\xi \rightarrow 0)$ - otherwise the equilibrium expressions would need to be modified to incorporate this non-infinitesimal fixed cost.
} 
that entry only takes place when it is strictly profitable. It is a useful starting point because it enables us to derive our main results in the most transparent way. In Section 4, we will explore what happens when the tie-breaking rule is changed, and show that this introduces the possibility of multiple equilibria.

We summarize the main results of this discussion in the following proposition.

Proposition 1 Let us impose the tie-breaking rule that whenever $w_{H}=w_{L}$, the incumbent fights in stage (ii) of the sequential entry-exit game. Then in any subgame-perfect equilibrium of the entry-and-exit game described above, there is only one active producer in equilibrium. When $w_{H}>w_{L}$, all hi-tech firms facing the entry of a low-tech competitor exit the market. When $w_{H} \leq w_{L}$ (where $w_{H}<w_{L}$ never occurs in equilibrium), hi-tech incumbents would fight entry, and no standardization occurs.

Since in equilibrium there is only one active producer, the price of each good will be a markup over the marginal cost:

$$
p_{H}=\left(1-\frac{1}{\epsilon}\right)^{-1} w_{H} \quad \text { and } \quad p_{L}=\left(1-\frac{1}{\epsilon}\right)^{-1} w_{L} .
$$

Symmetry and labor market clearing pin down the scale of production of each firm:

$$
x_{L}=\frac{L}{A_{L}} \text { and } x_{H}=\frac{H}{A_{H}}
$$

where recall that $H$ is the number of skilled workers employed by hi-tech firms and $L$ is the remaining labor force. Markup pricing implies that profits are a constant fraction of revenues:

$$
\pi_{H}=\frac{p_{H} H}{\epsilon A_{H}} \quad \text { and } \quad \pi_{L}=\frac{p_{L} L}{\epsilon A_{L}} .
$$

At this point, it is useful to define the following variables: $n \equiv A_{H} / A$ and $h \equiv H / L$. Here, $n$ is the fraction of hi-tech goods over the total and $h$ is the relative endowment of skilled workers. Then using demand (3) and (6), we can solve for relative prices as:

$$
\frac{p_{H}}{p_{L}}=\left(\frac{x_{H}}{x_{L}}\right)^{-1 / \epsilon}=\left(h \frac{1-n}{n}\right)^{-1 / \epsilon}
$$

and

$$
\frac{w_{H}}{w_{L}}=\frac{p_{H}}{p_{L}}=\left(h \frac{1-n}{n}\right)^{-1 / \epsilon} .
$$

Intuitively, the skill premium $w_{H} / w_{L}$ depends negatively on the relative supply of skill $(h=H / L)$ and positively on the relative number of hi-tech firms demanding skilled 
workers. Note that $w_{H}=w_{L}$ at:

$$
n^{\min } \equiv \frac{h}{h+1} .
$$

For simplicity, we restrict attention to initial states of technology such that $n>n^{\min }$. As an implication of Proposition 1, if we start from $n>n^{\min }$, the equilibrium will always remain in the interval $n \in\left[n^{\mathrm{min}}, 1\right]$. We can therefore restrict attention to this interval, over which skilled workers never seek employment in low-tech firms.

Using (7) and (8) yields relative profits:

$$
\frac{\pi_{H}}{\pi_{L}}=\left(h \frac{1-n}{n}\right)^{1-1 / \epsilon} .
$$

This equation shows that the relative profitability of hi-tech firms, $\pi_{H} / \pi_{L}$, is increasing in the relative supply of skill, $H / L$, because of a standard market size effect and decreasing in the relative number of hi-tech firm, $A_{H} / A_{L}$. The reason for the latter effect is that an increase in the number of firms of a given type increases the competition for the corresponding type of labor and reduces the equilibrium size of firms of that type.

Next, to solve for the level of profits, we first use symmetry into (4) to obtain:

$$
\begin{aligned}
& \frac{p_{H}}{A}=\left[(1-n)\left(\frac{p_{L}}{p_{H}}\right)^{1-\epsilon}+n\right]^{1 /(\epsilon-1)}, \text { and } \\
& \frac{p_{L}}{A}=\left[1-n+n\left(\frac{p_{H}}{p_{L}}\right)^{1-\epsilon}\right]^{1 /(\epsilon-1)} .
\end{aligned}
$$

Combining these expressions with (7) and (8) yields:

$$
\begin{aligned}
& \pi_{H}=\frac{H}{\epsilon}\left[1+\left(\frac{1}{n}-1\right)^{\frac{1}{\epsilon}} h^{\frac{1-\epsilon}{\epsilon}}\right]^{\frac{1}{\epsilon-1}} n^{\frac{2-\epsilon}{\epsilon-1}}, \text { and } \\
& \pi_{L}=\frac{L}{\epsilon}\left[1+\left(\frac{1}{n}-1\right)^{-\frac{1}{\epsilon}} h^{\frac{\epsilon-1}{\epsilon}}\right]^{\frac{1}{\epsilon-1}}(1-n)^{\frac{2-\epsilon}{\epsilon-1}}
\end{aligned}
$$

Note that, for a given $n$, profits per firm remain constant. The following lemma formalizes some important properties of the profit functions:

Lemma 1 Assume $\epsilon \geq 2$. Then, for $n \in\left[n^{\min }, 1\right]$ :

$$
\frac{\partial \pi_{H}}{\partial n}<0 \quad \text { and } \quad \frac{\partial \pi_{L}}{\partial n}>0 .
$$

Moreover, $\pi_{L}$ is a convex function of $n$. 
Proof. See the Appendix.

The condition $\epsilon \geq 2$ is sufficient - though not necessary - for the effect of competition for labor to be strong enough to guarantee that an increase in the number of hi-tech (low-tech) firms reduces the absolute profit of hi-tech (low-tech) firms. In the rest of the paper, we assume that the restriction on $\epsilon$ in Lemma 1 is satisfied. ${ }^{7}$

\subsection{Standardized Goods, Production and Profits}

Substituting (6) into (2), the equilibrium level of aggregate output can be expressed as:

$$
Y=A\left[(1-n)^{\frac{1}{\epsilon}} L^{\frac{\epsilon-1}{\epsilon}}+n^{\frac{1}{\epsilon}} H^{\frac{\epsilon-1}{\epsilon}}\right]^{\frac{\epsilon}{\epsilon-1}}
$$

showing that output is linear in the overall level of technology, $A$, and is a constantelasticity function of $H$ and $L$. From (14), we have that

$$
\frac{\partial Y}{\partial n}=\frac{A^{1-\frac{1}{\epsilon}} Y^{\frac{1}{\epsilon}}}{\epsilon-1}\left[\left(\frac{H}{n}\right)^{\frac{\epsilon-1}{\epsilon}}-\left(\frac{L}{1-n}\right)^{\frac{\epsilon-1}{\epsilon}}\right]
$$

which implies that aggregate output is maximized when $n /(1-n)=h$. Intuitively, production is maximized when the fraction of hi-tech products is equal to the fraction of skilled workers in the population, so that $x_{L}=x_{H}$ and prices are equalized across goods. Equation (14) is important in that it highlights the value of technology diffusion: by shifting some technologies to low-skill workers, standardization "alleviates" the pressure on scarce high-skill workers, thereby raising aggregate demand. It also shows that the effect of standardization on production, for given $A$, disappears as goods become more substitutable (high $\epsilon$ ). In the limit as $\epsilon \rightarrow \infty$, there is no gain to smoothing consumption across goods $\left(x_{L}=x_{H}\right)$ so that $Y$ only depends on aggregate productivity $A$.

Finally, to better understand the effect of technology diffusion on innovation, it is also useful to express profits as a function of $Y$. Using (2)-(4) to substitute $p_{H}=$ $A^{\frac{\epsilon-2}{\epsilon}}\left(Y / x_{H}\right)^{1 / \epsilon}$ into $(7)$, profits of a hi-tech firm can be written as:

$$
\pi_{H}=\frac{(Y / A)^{1 / \epsilon}}{\epsilon}\left(\frac{H}{n}\right)^{\frac{\epsilon-1}{\epsilon}} .
$$

A similar expression holds for $\pi_{L}$. Notice that profits are proportional to aggregate demand, $Y$. Thus, as long as faster technology diffusion (lower $n$ ) through standardization raises $Y$, it also tends to increase profits. On the contrary, an increase in $n \geq n^{\text {min }}$

\footnotetext{
${ }^{7}$ An elasticity of substitution between products greater than 2 is consistent with most empirical evidence in this area. See, for example, Broda and Weinstain (2006).
} 
reduces the instantaneous profit rate of hi-tech firms, i.e.,

$$
\frac{\partial \pi_{H}}{\partial n} \frac{n}{\pi_{H}}=\frac{1}{\epsilon} \frac{\partial Y}{\partial n} \frac{n}{Y}-\frac{\epsilon-1}{\epsilon}<0
$$

\subsection{InNOVATion AND StandardizATion}

We model both innovation, i.e., the introduction of a new hi-tech good, and standardization, i.e., the process that turns an existing hi-tech product into a low-tech variety, as costly activities. We follow the "lab-equipment" approach and define the costs of these activities in terms of output, $Y$. In particular, we assume that introducing a new hi-tech good requires $\mu_{H}$ units of the numeraire, while standardizing an existing hi-tech good costs $\mu_{L}$ units of $Y$. We may think of $\mu_{L}$ as capturing the technical cost of simplifying the production process plus any policy induced costs due to IPR regulations restricting the access to new technologies.

Next, we define $V_{H}$ and $V_{L}$ as the net present discounted values of firms hi-tech and low-tech goods, respectively. These are given by the discounted value of the expected profit stream earned by each type of firm and must satisfy the following HamiltonJacobi-Bellman equations:

$$
\begin{aligned}
r V_{L} & =\pi_{L}+\dot{V}_{L} \\
r V_{H} & =\pi_{H}+\dot{V}_{H}-m V_{H}
\end{aligned}
$$

where $m$ is the arrival rate of standardization, which is endogenous and depends on the intensity of investment in standardization. These equations require that the instantaneous profit from running a firm plus any capital gain or losses must be equal to the return from lending the market value of the firm at the risk-free rate, $r$. Note that, at a flow rate $m$, a hi-tech firm is replaced by a low-tech producer and the value $V_{H}$ is lost.

Free-entry in turn implies that the value of innovation and standardization can be no greater than their respective costs:

$$
V_{H} \leq \mu_{H} \quad \text { and } \quad V_{L} \leq \mu_{L}
$$

If $V_{H}<\mu_{H}\left(V_{L}<\mu_{L}\right)$, then the value of innovation (standardization) is lower than its cost and there will be no investment in that activity.

\subsection{Dynamic Equilibrium}

A dynamic equilibrium is a time path for $\left(C, x_{i}, A, n, r, p_{i}\right)$ such that monopolists maximize the discounted value of profits, the evolution of technology is determined by free entry in innovation and standardization, the time path for prices is consistent 
with market clearing and the time path for consumption is consistent with household maximization. We will now show that a dynamic equilibrium can be represented as a solution to two differential equations. Let us first define:

$$
\chi \equiv \frac{C}{A} ; \quad y \equiv \frac{Y}{A} ; \quad g \equiv \frac{\dot{A}}{A} .
$$

The first differential equation is the law of motion of the fraction of hi-tech goods, $n$. This is the state variable of the system. Given that hi-tech goods are replaced by a low-tech goods at the endogenous rate $m$, the flow of newly standardized products is $\dot{A}_{L}=m A_{H}$. From this and the definition $n=\left(A-A_{L}\right) / A$ we obtain:

$$
\dot{n}=(1-n) g-m n .
$$

The second differential equation is the law of motion of $\chi$. Differentiating $\chi$ and using the consumption Euler equation (1) yields:

$$
\frac{\dot{\chi}}{\chi}=r-\rho-g
$$

Next, to solve for $g$, we use the aggregate resource constraint. In particular, consumption is equal to production minus investment in innovation, $\mu_{H} \dot{A}$, and in standardization, $\mu_{L} \dot{A}_{L}$. Noting that $\dot{A} / A=g$ and $\dot{A}_{L} / A=m n$, we can thus write:

$$
\chi=y-\mu_{H} g-\mu_{L} m n
$$

Substituting for $g$ from this equation (i.e., $\left.g=\left(y-\mu_{L} m n-\chi\right) / \mu_{H}\right)$ into (19) and (20) gives the following two equation dynamical system in the $(n, \chi)$ space:

$$
\begin{gathered}
\frac{\dot{\chi}}{\chi}=r-\rho-\frac{y-\mu_{L} m n-\chi}{\mu_{H}} \\
\frac{\dot{n}}{n}=\left(\frac{1-n}{n}\right) \frac{y-\chi}{\mu_{H}}-m\left(1+(1-n) \frac{\mu_{L}}{\mu_{H}}\right),
\end{gathered}
$$

Note that $y$ is a function of $n$ (see equation (14)). Finally, $r$ and $m$ can be found as functions of $n$ from the Hamilton-Jacobi-Bellman equations. First, note that, if there is positive imitation $(m>0)$, then free-entry implies $V_{L}=\mu_{L}$. Given that $\mu_{L}$ is constant, $V_{L}$ must be constant too, $\dot{V}_{L}=0$. Likewise, if there is positive innovation $(g>0)$, then $\dot{V}_{H}=0$. Next, equations (18) can be solved for the interest rate in the two cases:

$$
r=\frac{\pi_{L}}{\mu_{L}} \quad \text { if } \quad m>0
$$




$$
r=\frac{\pi_{H}}{\mu_{H}}-m \quad \text { if } \quad g>0
$$

We summarize these findings in the following proposition.

Proposition 2 A dynamic equilibrium is characterized by (i) the autonomous system of differential equations (21)-(22) in the $(n, \chi)$ space where

$$
\begin{aligned}
& y=y(n)=\left[(1-n)^{\frac{1}{\epsilon}} L^{\frac{\epsilon-1}{\epsilon}}+n^{\frac{1}{\epsilon}} H^{\frac{\epsilon-1}{\epsilon}}\right]^{\frac{\epsilon}{\epsilon-1}}, \\
& r=r(n)=\max \left\{\frac{\pi_{L}(n)}{\mu_{L}}, \frac{\pi_{H}(n)}{\mu_{H}}-m\right\}, \\
& m=m(n)=\left\{\begin{array}{lll}
0 & \text { if } r>\frac{\pi_{L}(n)}{\mu_{L}} \\
\frac{y(n)-\chi}{n \mu_{L}} & \text { if } r>\frac{\pi_{H}(n)}{\mu_{H}}-m
\end{array},\right.
\end{aligned}
$$

and $\pi_{H}(n)$ and $\pi_{L}(n)$ are given by (12); (ii) a pair of initial conditions, $n_{0}$ and $A_{0}$; and (iii) the transversality condition $\lim _{t \rightarrow \infty}\left[\exp \left(-\int_{0}^{t} r_{s} d s\right) \int_{0}^{A_{t}} V_{i} d i\right]=0$.

\subsection{Balanced Growth Path}

A Balanced Growth Path (BGP) is a dynamic equilibrium such that $\dot{n}=\dot{m}=0$. In BGP, the skill premium and the interest rate are at a steady-state level. An "interior" BGP is a BGP where, in addition, $m>0$ and $g>0$. Equation (19) implies that an interior BGP must feature $m^{s s}=g(1-n) / n=(r-\rho)(1-n) / n$. To find the associated BGP interest rate, we use the free-entry conditions for standardization and innovation. Using (12), the following equation determines the interest rate consistent with $m>0$ :

$$
\begin{aligned}
r_{L}(n) & \equiv \frac{\pi_{L}}{\mu_{L}} \\
& =\frac{L}{\mu_{L} \epsilon}\left[1+\left(\frac{1}{n}-1\right)^{-\frac{1}{\epsilon}} h^{\frac{\epsilon-1}{\epsilon}}\right]^{\frac{1}{\epsilon-1}}(1-n)^{\frac{2-\epsilon}{\epsilon-1}}
\end{aligned}
$$

Next, the free-entry condition for hi-tech firms, conditional on the BGP standardization rate, determines the interest rate consistent with the BGP:

$$
\begin{aligned}
r_{H}^{s s}(n) & \equiv \frac{\pi_{H}}{\mu_{H}}-m^{s s}=n \frac{\pi_{H}}{\mu_{H}}+(1-n) \rho \\
& =\frac{H}{\mu_{H} \epsilon}\left[1+\left(\frac{1}{n}-1\right)^{\frac{1}{\epsilon}} h^{\frac{1-\epsilon}{\epsilon}}\right]^{\frac{1}{\epsilon-1}} n^{\frac{1}{\epsilon-1}}+(1-n) \rho .
\end{aligned}
$$

The curves $r_{H}^{s s}(n)$ and $r_{L}(n)$ can be interpreted as the (instantaneous) return from innovation (conditional on $\dot{n}=0$ ) and standardization, respectively. In the space $(n, r)$, 
the BGP value of $n$ can be found as the crossing point of these curves: in other words, along a BGP, both innovation and standardization must be equally profitable. ${ }^{8}$ We summarize the preceding discussion in the following proposition:

Proposition 3 An interior BGP is a dynamic equilibrium such that $n=n^{\text {ss }}$ where $n^{\text {ss }}$ satisfies

$$
r_{L}\left(n^{s s}\right)=r_{H}^{s s}\left(n^{s s}\right),
$$

and $r_{L}\left(n^{s s}\right)$ and $r_{H}^{s s}\left(n^{s s}\right)$ are given by (25) and (26), respectively. Given $n^{s s}$, the BGP interest rate is $r^{s s}=\pi_{L}\left(n^{s s}\right) / \mu_{L}$, and the standardization rate is $m^{s s}=\left(r^{s s}-\rho\right)\left(1-n^{s s}\right) / n^{s s}$, where $\pi_{L}$ is as in (12) [evaluated at $n=n^{s s}$ ]. Finally, $A_{H}, A_{L}, Y$ and $C$ all grow at the same rate, $g^{s s}=r^{s s}-\rho$.

To characterize the set of BGPs, we need to study the properties of $r_{L}(n)$ and $r_{H}^{s s}(n)$. Due to the shape of $\pi_{L}, r_{L}(n)$ is increasing and convex. Provided that $\rho$ is not too high, $r_{H}^{s s}(n)$ is non-monotonic (first increasing and then decreasing) and concave in $n .^{9}$ The intuition for the non monotonicity is as follows. When $n$ is high, competition for skilled workers among hi-tech firms brings $\pi_{H}$ down and this lowers the return to innovation. Moreover, when $n$ is higher than $h /(h+1)$, aggregate productivity and $Y$ are low, because skilled workers have too many tasks to perform, while unskilled workers too little. This tends to reduce $\pi_{H}$ even further. When $n$ is low, $\pi_{H}$ is high, but the flow rate of standardization is high as well (since, recall, $m^{s s}=g(1-n) / n$ ) and this brings down the return to innovation.

Figure 1 shows the BGP relationship between $r$ and $n$. Note that, as long as $m>0$, the equilibrium must lie on the (dashed) $r_{L}(n)$ curve. An interior BGP must also lie on the (solid) $r_{H}^{s s}(n)$ curve. Thus, the interior BGP value of $n$ is identified by their intersection. The following assumptions guarantee the existence and uniqueness of a $\mathrm{BGP}$, and that such BGP is interior.

Assumption $10<\rho<H /\left(\mu_{H} \epsilon\right)$.

This condition is standard: it guarantees that innovation is sufficiently profitable to sustain endogenous growth and that the transversality condition is satisfied.

Assumption $2 \mu_{H}<\mu_{L} \frac{h}{1+h-\epsilon \rho \mu_{L} /(H+L)}$.

Assumption 2 ensures that $r_{H}^{s s}\left(n^{\min }\right)>r_{L}\left(n^{\min }\right)$, ruling out the uninteresting case in which standardization is always more profitable than innovation when $n$ is expected to stay constant, and guarantees that the BGP is interior and unique. We state the existence and uniqueness of the BGP in a formal proposition.

\footnotetext{
${ }^{8}$ It can also be verified straightforwardly that the allocation corresponding to this crossing point satisfies the transversality condition.

${ }^{9} \mathrm{~A}$ formal argument can be found in the proof of Proposition 4.
} 


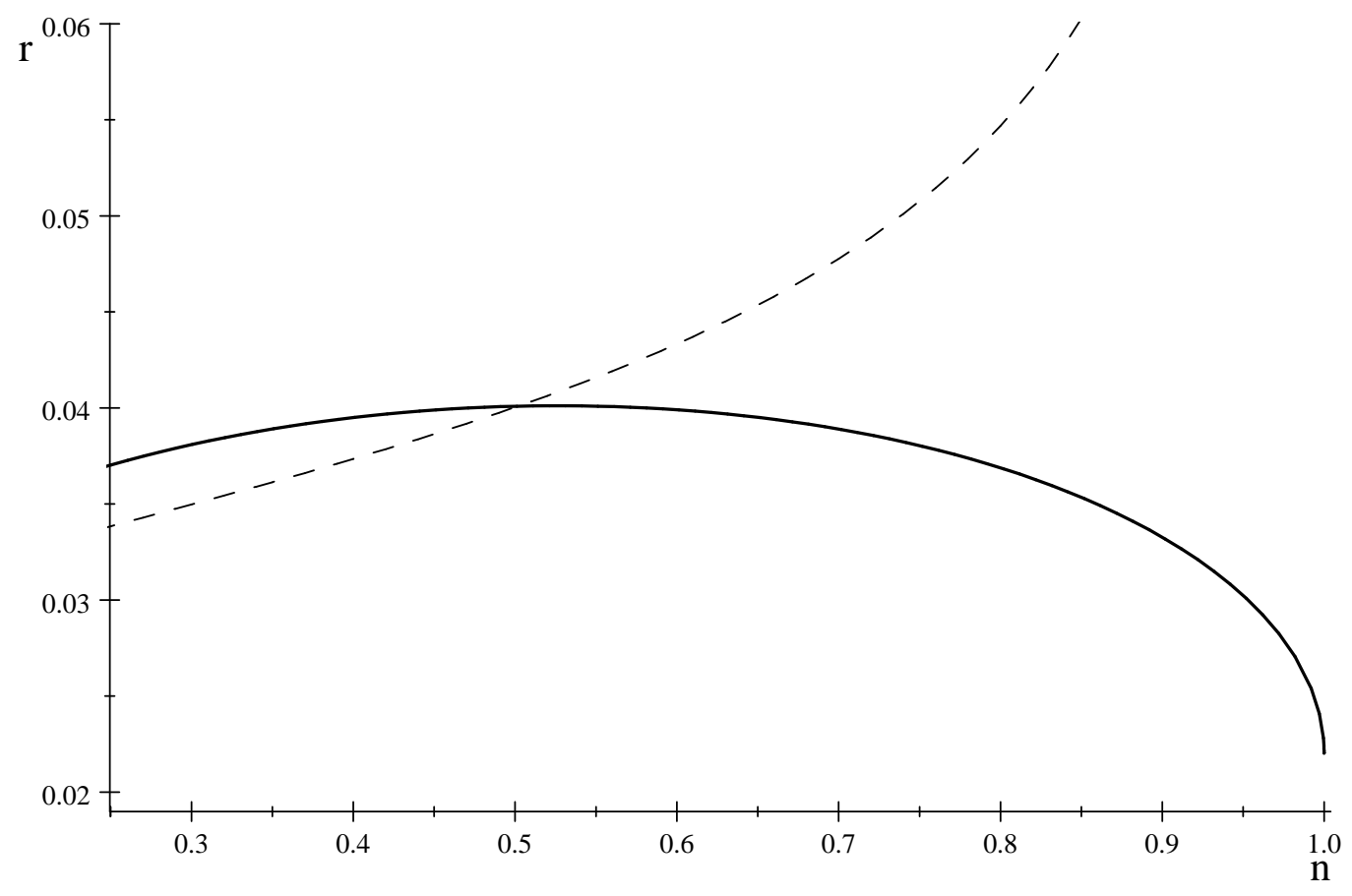

Figure 1: Solid $=r_{H}^{s s}(n)$, Dashed $=r_{L}(n)$

Proposition 4 Suppose Assumptions 1-2 hold. Then there exist a unique BGP equilibrium.

Proof. See the Appendix.

Proposition 4 establishes the existence and uniqueness of a BGP equilibrium, denoted by $\left(n^{s s}, \chi^{s s}\right)$. Our next goal is to prove the (local) existence and uniqueness of a dynamic equilibrium converging to this BGP. Unfortunately, the analysis of dynamics is complicated by several factors. First, the dynamical system (21)-(22) is highly nonlinear. Second, it may exhibit discontinuities in the standardization rate (and thus in the interest rate) along the equilibrium path. Intuitively, at $\left(n^{s s}, \chi^{s s}\right)$ there is both innovation and standardization (otherwise we could not have $\dot{n}=0$ ). It is relatively easy to prove that, similar to models of directed change (e.g., Acemoglu 2002 and Acemoglu and Zilibotti 2001), there exists a dynamic equilibrium converging to the BGP featuring either only innovation (when $n<n^{s s}$ ) or only standardization (when $n>n^{s s}$ ). This implies that when the economy approaches the BGP from the left, the standardization rate and the interest rate both jump once the BGP is reached. In particular, when $n<n^{s s}$, there is no standardization, thus, $m=0$, while in BGP we have $m>0$. Since throughout there is innovation, the value of hi-tech firms must remain constant at $V_{H}=\mu_{H}$ and thus there can be no jump in $r+m$. Consequently, there must be an exactly offsetting jump 
in interest rate $r$ when the BGP is reached and the standardization rate, $m$, jumps. ${ }^{10}$

However, it turns out to be more difficult to prove that there exist no other dynamic equilibria. In particular, we must rule out the existence of equilibrium trajectories (solutions to (21)-(22)) converging to $\left(n^{s s}, \chi^{s s}\right)$ with both innovation and standardization when the economy is away from the BGP. Numerical analysis suggests that no such trajectory exists as long as Assumptions 1 and 2 are satisfied. In particular, the system (21)-(22), under the condition that $m=\pi_{H}(n) / \mu_{H}-\pi_{L}(n) / \mu_{L}$ (i.e., under the condition that there is both innovation and standardization), is globally unstable around $\left(n^{s s}, \chi^{s s}\right)$. However, we can only prove this analytically under additional conditions. In particular, we must impose the following parameter restriction: ${ }^{11}$

$$
\frac{\epsilon-1}{\epsilon}(h+2)+\epsilon>\frac{2 h+1}{h(h+1)} .
$$

Proposition 5 Suppose that Assumptions 1 and 2 and (28) hold. Then there exists $\bar{\rho}>0$ such that, for $\rho<\bar{\rho}$, the interior BGP is locally saddle-path stable. In particular, if $n_{t_{0}}$ is in the neighborhood of its BGP value, $n^{s s}$, and $n_{t_{0}}>n^{s s}\left[\right.$ resp., $\left.n_{t_{0}}<n^{s s}\right]$, then there exists a unique path converging to the BGP such that for some finite $\bar{t}>t_{0}$, we have $\tau \in\left[t_{0}, \bar{t}\right], m_{\tau}>0, g_{\tau}=0$ and $\dot{n}_{\tau}<0$ [resp., $m_{\tau}=0, g_{\tau}>0$ and $\dot{n}_{\tau}>0$ ], and the economy attains the BGP at $\bar{t}$ (i.e., for all $\tau \geq \bar{t}$, we have $n_{\tau}=n^{s s}, m_{\tau}=m^{s s}$, and $\left.g_{\tau}=g^{s s}\right)$.

\section{Proof. See the Appendix.}

\subsection{Growth and Standardization: an Inverse-U Relationship}

How does the cost of standardization, $\mu_{L}$, affect the BGP growth rate, $g^{s s}$ ? Answering this question is important from both a normative and a positive perspective. First, policies such as IPR protection are likely to have an impact on the profitability of standardization. Therefore, knowing the relationship between standardization and growth is a key step for policy evaluation. Second, the difficulty of standardization and hence its costs may vary across technologies and over time.

The cost of standardization affects $r_{L}(n)$, but not $r_{H}^{s s}(n)$. Thus, increasing the cost of standardization amounts to shifting the $r_{L}(n)$ curve in Figure 1 and therefore the intersection, form $n=n^{\text {min }}\left(\right.$ low $\left.\mu_{L}\right)$ to $n \rightarrow 1$ (high $\left.\mu_{L}\right)$. The effect on the growth rate

\footnotetext{
${ }^{10}$ Note that the discontinuous behavior of the standardization rate and the interest rate does not imply a jump in the asset values, $V_{H}$ and/or $V_{L}$. Rather, the rate of change of these asset values may jump locally.

${ }^{11}$ This restriction, which ensures that $n_{\min }=h /(1+h)$ is not too small, is used in the Appendix. For example, when $\epsilon=2$, it requires $n_{\min }=h /(1+h)>0.28$ and when $\epsilon=3, n_{\min }=h /(1+h)>0.21$.
} 
depends in turn on the relationship between $g^{s s}$ and $n$ :

$$
g^{s s}(n)=r_{H}^{s s}(n)-\rho=n\left(\frac{\pi_{H}(n)}{\mu_{H}}-\rho\right) .
$$

This expression highlights the trade-off between innovation and standardization: a high standardization rate (and thus a low $n$ ) increases the instantaneous profit rate $\pi_{H}(n)$, but lowers the expected profit duration. Taking the derivative and using (17) yields:

$$
\begin{aligned}
\frac{\partial g^{s s}(n)}{\partial n} & =\frac{\pi_{H}(n)}{\mu_{H}}-\rho+\frac{n}{\mu_{H}} \frac{\partial \pi_{H}(n)}{\partial n} \\
& =\frac{\pi_{H}(n)}{\epsilon \mu_{H}}\left(1+\frac{\partial Y(n)}{\partial n} \frac{n}{Y(n)}\right)-\rho
\end{aligned}
$$

From (15), $\partial Y(n) / \partial n=0$ at $n=n^{\text {min }}$. For $n>n^{\text {min }}$, we have $\partial Y(n) / \partial n<0$ with $\lim _{n \rightarrow 1} \partial Y(n) / \partial n=-\infty$. Thus:

$$
\left.\frac{\partial g^{s s}(n)}{\partial n}\right|_{n=n^{\min }}=\frac{H+L}{\mu_{H} \epsilon^{2}}-\rho \text { and } \quad \lim _{n \rightarrow 1} \frac{\partial g^{s s}(n)}{\partial n}=-\infty .
$$

Provided that $\rho<\frac{H+L}{\mu_{H} \epsilon^{2}}, g^{s s}(n)$ is an inverse- $\mathrm{U}$ function of $n$. Intuitively, at $n=1$ the wage of unskilled workers is zero and hence the marginal value of transferring technologies to them (in terms of higher aggregate demand and thus also profits) is infinite. Instead, at $n=n^{\text {min }}$, aggregate output $Y$ is maximized and marginal changes in $n$ have second order effects on aggregate production. Moreover, given that future profits are discounted, the impact of prolonging the expected profit stream (high $n$ ) on innovation vanishes if $\rho$ is high. When $\rho<\frac{H+L}{\mu_{H} \epsilon^{2}}$, growth is maximized at $n^{*} \in\left(n^{\mathrm{min}}, 1\right)$ that solves:

$$
1-\rho \frac{\epsilon \mu_{H}}{\pi_{H}\left(n^{*}\right)}=-\frac{\partial Y\left(n^{*}\right)}{\partial n^{*}} \frac{n^{*}}{Y\left(n^{*}\right)}
$$

The condition $\rho<\frac{H+L}{\mu_{H} \epsilon^{2}}$ is satisfied whenever Assumption 1 (which we imposed above and which guarantees $g>0$ ) and $\epsilon<1+1 / h$, i.e., if skilled workers are sufficiently scarce. It is also satisfied when $\rho$ and $\mu_{H}$ are sufficiently low. Now recalling that in BGP $n$ is an increasing function of $\mu_{L}$, we have the following result (proof in the text):

Proposition 6 Let $g^{s s}$ be the BGP growth rate and suppose that $\rho<\frac{H+L}{\mu_{H} \epsilon^{2}}$. Then, $g^{s s}$ is an inverse $U$-shaped function of the cost of standardization.

Figure 1 provides a geometric intuition. Starting from a very high $\mu_{L}$ such that $r_{H}^{s s}(n)$ is in its decreasing portion, a decrease in $\mu_{L}$ moves the equilibrium to the left along the schedule $r_{H}^{s s}(n)$. This yields a lower $n^{s s}$ and thus higher growth. Therefore, in this region, a decrease in $\mu_{L}$ increases growth. However, after the maximum of the $r_{H}^{s s}(n)$ schedule is passed, further decreases in $\mu_{L}$ reduce $n$ and growth. 


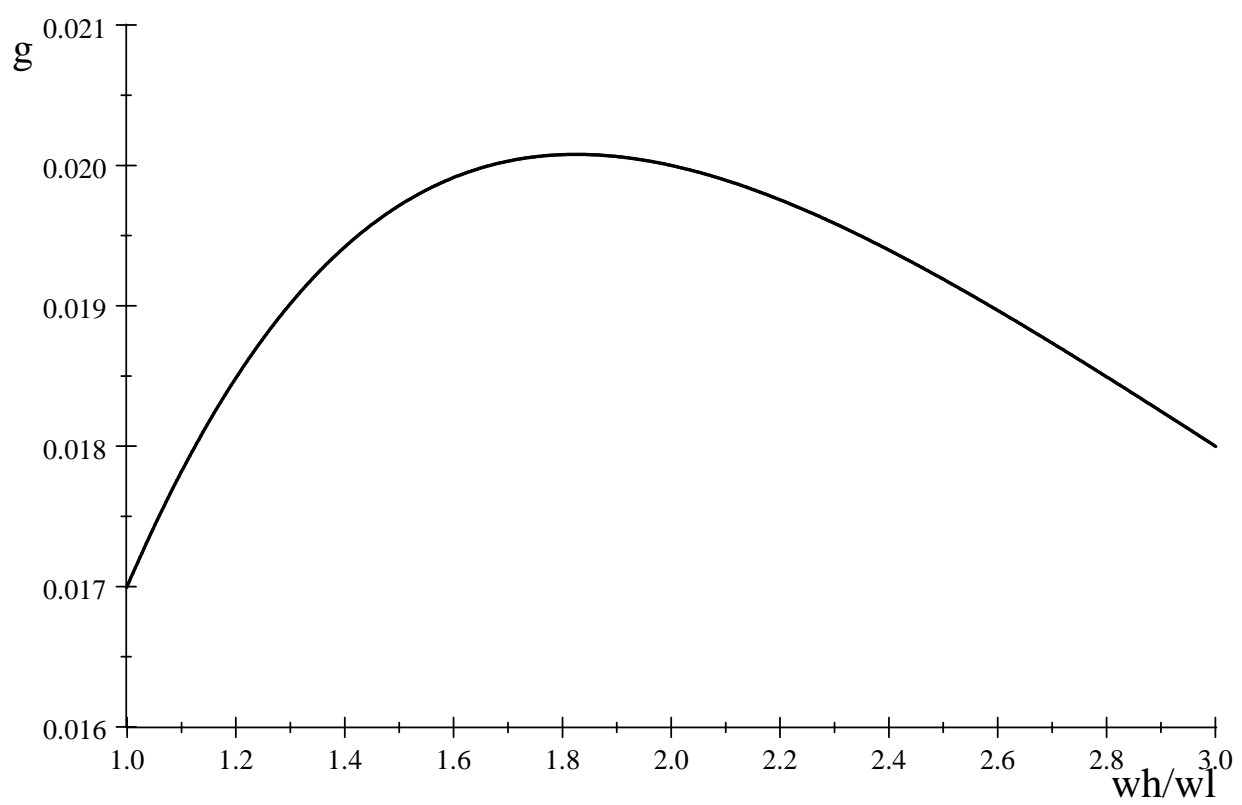

Figure 2: Growth and the Skill Premium

Proposition 6 also has interesting implications for the skill premium. Recall that, in this model, the skill-premium is the market value of being able to operate new technologies and produce hi-tech goods. For this reason, it is increasing in the fraction of hi-tech firms (see equation (9)). Since growth is an inverse-U function of $n$, the model also predicts a inverse U-shaped relationship between growth and wage inequality, as shown in Figure 2. Intuitively, a very high skill-premium could be a sign that standardization is so costly that growth has slowed down. A very low skill premium, on the other hand, might be a sign of excessive standardization, weakening innovation incentives and slowing down growth.

\section{Welfare Analysis and Optimal Policies}

We now turn to the normative analysis. We start by characterizing the Pareto optimal allocation for a given $\mu_{L}$, representing the technical cost of standardization. This allows us to identify the inefficiencies that are present in the decentralized equilibrium. Next, we focus on the constrained efficient allocation that a government could achieve with a limited set of instruments. In particular, we allow the government to increase the cost of standardization above $\mu_{L}$ through IPR regulations and to influence the level of competition. Finally, we briefly discuss how North-South trade affects the optimal policies. 


\subsection{Pareto Optimal Allocation}

The Pareto optimal allocation is the one chosen by a social planner seeking to maximize the utility of the representative agent, subject to the production function (14) and for given costs of innovation, $\mu_{H}$, and standardization, $\mu_{L}$. The current value Hamiltonian for the problem is:

$$
\mathcal{H}=\ln \left(Y-I_{H}-I_{L}\right)+\xi_{H} \frac{I_{H}}{\mu_{H}}+\xi_{L} \frac{I_{L}}{\mu_{L}}
$$

where $I_{H}$ and $I_{L}$ are investment in innovation and standardization, respectively. The control variables are $I_{H}$ and $I_{L}$, while the state variables are $A$ and $A_{L}$, with co-state variables $\xi_{H}$ and $\xi_{L}$, respectively. From the first order conditions, the Pareto optimal $n$ solves:

$$
\frac{\partial Y}{\partial A} \frac{1}{\mu_{H}}=\frac{\partial Y}{\partial A_{L}} \frac{1}{\mu_{L}}
$$

That is, the planner equates the marginal rate of technical substitution between hi-tech and low-tech products to their relative development costs. The Euler equation for the planner is:

$$
\frac{\dot{C}}{C}=\frac{\partial Y}{\partial A} \frac{1}{\mu_{H}}-\rho .
$$

By comparing these results to those in the previous section, we can see that the decentralized equilibrium is inefficient for two reasons.

First, there is a standard appropriability problem whereby firms only appropriate a fraction of the value of innovation/standardization so that $\mathrm{R} \& \mathrm{D}$ investment is too low. To isolate this inefficiency, consider the simplest case $L=0$, so that there is no standardization and $Y=A H, \pi_{H}=H / \epsilon$. In this case, the social return from innovation is $H$ while the private return is only $r=H / \epsilon<H$. The same form of appropriability effect also applies when $L>0$.

Second, there is too much standardization relative to innovation due to a business stealing externality: the social value of innovation is permanent while the private benefit is temporary. A particularly simple case to highlight this inefficiency is when $\epsilon=2$ so that (30) simplifies to:

$$
\frac{n}{1-n}=h\left(\frac{\mu_{L}+\mu_{H}}{\mu_{H}}\right)^{2},
$$

In the decentralized equilibrium, instead, the condition $r_{L}(n)=r_{H}^{s s}(n)$ yields:

$$
\frac{n}{1-n}=h\left[\left(\frac{r}{m+r}\right) \frac{\mu_{L}}{\mu_{H}}\right]^{2}
$$

Clearly, $n$ is too low in the decentralized economy.

To correct the first inefficiency, subsidies to innovation (and standardization) are needed. On the other hand, the business stealing externality can be corrected by intro- 
ducing a licensing policy requiring low-tech firms to compensate the losses they impose on hi-tech firms. In particular, suppose that firms that standardize must pay a one-time licensing fee $\mu_{L}^{l i c}$ to the original inventor. In this case, the free-entry conditions together with the Hamilton-Jacobi-Bellman equations (18) become:

$$
\begin{aligned}
V_{L} & =\frac{\pi_{L}}{r}=\mu_{L}^{l i c}+\mu_{L} \\
V_{H} & =\frac{\pi_{H}-m\left(V_{H}-\mu_{L}^{l i c}\right)}{r}=\mu_{H} .
\end{aligned}
$$

Clearly, the business stealing effect is removed when $\mu_{L}^{l i c}=\mu_{H}$, that is, when low-tech firms compensate the hi-tech produces for the entire capital loss $\mu_{H}$. We summarize these results in the proposition (proof in the text). ${ }^{12}$

Proposition 7 The Pareto optimal allocation can be decentralized using a subsidy to innovation and a license fee imposed on firms standardizing new products.

\subsection{Constrained Efficiency: Optimal $\mu_{L}$}

Proposition 7 shows how the Pareto optimal allocation can be decentralized. However, the subsidies to innovation require lump-sum taxes and in addition, the government would need to set up and operate a system of licensing fees. In practice, both of these might be difficult. ${ }^{13}$ Motivated by this reasoning, we now analyze a constrained efficient policy, where we limit the instruments of the government. In particular, we assume that the government can only affect the standardization cost through IPR regulations restricting the access to new technologies, and ask what would the optimal policy be in

\footnotetext{
${ }^{12}$ Note that there is no static distortion due to monopoly pricing. This is because in our model all firms only use inelastically supplied factors (skilled and unskilled labor). Thus, markups do not distort the allocation. In a more general model, subsidies to production would also be needed correct for the static inefficiency.

${ }^{13}$ Licensing may fail in practice because of incompleteness of contracts or because of asymmetric information (e.g., Bessen and Maskin, 2009). See also Chari et al. (2009) on the difficulties of using market signals to determine the value of existing innovations.
} 
this case. ${ }^{14}$ More precisely, we find the (constant) $\mu_{L}^{*}$ that maximizes BGP utility:

$$
\begin{aligned}
\max _{\mu_{L}} \rho W & =\rho \int_{0}^{\infty}\left[\ln \left(\frac{C}{A}\right)+\ln \left(A_{0} e^{g t}\right)\right] e^{-\rho t} d t \\
& =\ln \left(\chi^{s s}\right)+\frac{g^{s s}}{\rho} .
\end{aligned}
$$

The optimal policy maximizes a weighted sum of the consumption level and its growth rate. In turn, $g^{s s}\left(\mu_{L}\right)=n\left[\pi_{H}(n) / \mu_{H}-\rho\right]$ evaluated at the $n\left(\mu_{L}\right)$ that solves (27) and $\chi^{s s}=y(n)-g\left(\mu_{L}\right)\left[\mu_{H}+\mu_{L}(1-n)\right]$, evaluated at the same $n$. In general, problem (31) does not have a closed-form solution. Nonetheless, we can make progress by considering two polar cases.

\subsubsection{Optimal/Growth Maximizing Policy: $\rho \rightarrow 0$}

As $\rho \rightarrow 0$, the optimal policy is to maximize $g^{s s}$. For this case, we have simple analytic results. Manipulating the first order condition (29), the optimal $n^{*}$ is implicitly defined by:

$$
\left(\frac{1-n^{*}}{n^{*}} h\right)^{\frac{\epsilon-1}{\epsilon}}=1-\frac{1}{n^{*}}\left(1-\frac{1}{\epsilon}\right)
$$

Note that the LHS is decreasing in $n$, from infinity to zero, while the RHS is increasing in $n$, ranging from minus infinity to $1 / \epsilon$. Thus, the solution $n^{*}$ is always interior and unique. Using the implicit function theorem yields:

$$
\frac{\partial n^{*}}{\partial \epsilon} \frac{\epsilon}{n^{*}}=\frac{\epsilon\left(1-n^{*}\right)}{\epsilon-1}>0 \quad \text { and } \quad \frac{\partial n^{*}}{\partial h} \frac{h}{n^{*}}=\left(1-n^{*}\right)\left(1-\epsilon+n^{*} \epsilon\right)>0
$$

because, from (32), $\epsilon n^{*}-\epsilon+1=\epsilon\left(n^{*}\right)^{\frac{1}{\epsilon}}\left(1-n^{*}\right)^{\frac{\epsilon-1}{\epsilon}} h^{\frac{\epsilon-1}{\epsilon}}>0$. That is, the optimal fraction of hi-tech goods is increasing in the relative skill-endowment and in the elasticity of substitution across products.

Once we have $n^{*}$, we can use the indifference condition between innovation and standardization, $\frac{V_{H}}{V_{L}}=\frac{\mu_{H}}{\mu_{L}}$, to solve for the $\mu_{L}^{*}$ that implements $n^{*}$. When $\rho \rightarrow 0$ and

\footnotetext{
${ }^{14}$ We do not consider patent policies explicitly because we view them as only one source of competitive advantage for incumbents. In particular, patents are generally thought to be less important than lead time and learning-curve advantage in preventing duplication. Their utility might often be limited by the fact that they require disclosure of information, which would otherwise remain trade secret, and the application process is often lengthy and cannot prevent competitors from "inventing around" patents. Overall, Levin et al. (1987) found that patents increase imitation costs by 7-15\%. This supports both our approach of modeling IPR protection as an additional cost of standardization and the notion that patents are only one of the many factors making standardization costly.
} 
$m=g(1-n) / n$ we obtain:

$$
\frac{\pi_{H}}{\pi_{L}}=\frac{\mu_{H}}{\mu_{L}} \frac{1}{n} \rightarrow\left(\frac{1-n}{n} h\right)^{\frac{\epsilon-1}{\epsilon}}=\frac{\mu_{H}}{\mu_{L}} \frac{1}{n}
$$

The equilibrium fraction of hi-tech goods, $n$, depends on relative profits $\left(\pi_{H} / \pi_{L}\right)$, relative R\&D costs $\left(\mu_{H} / \mu_{L}\right)$, and the standardization risk faced by hi-tech firms (captured by the factor $1 / n)$. Note that a decline in the relative skill supply, $h$, leads to a more than proportional fall in $n$ because $\pi_{H} / \pi_{L}$ falls and $m$ rises. Substituting $\left(\frac{1-n^{*}}{n^{*}} h\right)^{\frac{\epsilon-1}{\epsilon}}$ from (32) we can find the optimal standardization cost as:

$$
\mu_{L}^{*}=\mu_{H}\left(n^{*}-1+\frac{1}{\epsilon}\right)^{-1}=\frac{\mu_{H} \epsilon}{1-\epsilon+n^{*} \epsilon} .
$$

This expression has the advantage that it only depends on $h$ through $n^{*}$ and can be used to study the determinants of the optimal policy. Differentiating (35) and using (33), we obtain the following proposition (proof in the text).

Proposition 8 Consider the case $\rho \rightarrow 0$. BGP welfare and growth are maximized when the cost of standardization $\mu_{L}$ satisfies (35) and $n^{*}$ is the solution to (32). The following comparative static results hold:

$$
\begin{aligned}
\frac{\partial \mu_{L}^{*}}{\partial \mu_{H}} \frac{\mu_{H}}{\mu_{L}^{*}} & =1 \\
\frac{\partial \mu_{L}^{*}}{\partial h} \frac{h}{\mu_{L}^{*}} & =-\epsilon n^{*}\left(1-n^{*}\right)<0 \\
\frac{\partial \mu_{L}^{*}}{\partial \epsilon} \frac{\epsilon}{\mu_{L}^{*}} & =\frac{n^{*} \epsilon-1}{\epsilon-1}>0 .
\end{aligned}
$$

The results summarized in this proposition are intuitive. Changes in the cost of innovation should be followed by equal changes in the cost of standardization, so as to keep the optimal $n$ constant. A decline in the relative supply of skilled workers makes technology diffusion relatively more important. However, the incentive to standardize increases so much (both because of the change in instantaneous profits and because the equilibrium $m$ increases too) that the optimal policy is to make standardization more costly. Thus, somewhat surprisingly, a higher abundance of unskilled worker calls for stronger IPR protection. Finally, given that $\epsilon \geq 2$, a lower elasticity of substitution makes the diffusion of technologies to low-skill workers more important for aggregate productivity and reduces the optimal IPR, $\mu_{L}^{*} \cdot{ }^{15}$

\footnotetext{
${ }^{15}$ To see this, recall that $\epsilon n^{*}-\epsilon+1>0$. Then, $\epsilon \geq 2$ implies $\left(\epsilon n^{*}-1\right) /(\epsilon-1)>0$.
} 


\subsubsection{Optimal Policy: high $\rho$}

To understand how the optimal policy changes with the discount rate, we consider the other polar case. In particular, assume that $\rho \rightarrow \frac{H+L}{\mu_{H} \epsilon}$. As we will see, this is the highest $\rho$ compatible with positive growth. In this case, Section 2.7 shows that $g$ is maximized at the corner $n^{\min }=h /(h+1)$. Moreover, for $n \rightarrow n^{\min }$ we have $\pi_{H}=\frac{L+H}{\epsilon}$ and $g=\frac{H+L}{\mu_{H} \epsilon}-\rho \rightarrow 0$ (since $\rho \rightarrow \frac{H+L}{\mu_{H} \epsilon}$ ). Next, the result that $g$ must be close to zero yields $\chi^{s s}=y$, which is also maximized at $n^{\text {min }}$. Thus, with high discounting the optimal policy is the same as the one that maximizes static output (and consumption) only. Reaching this point requires setting $\mu_{L}^{*}=\mu_{H}$. Note that, in this extreme scenario, the optimal policy becomes independent of $h$ and other parameters. Comparing the policy $\mu_{L}^{*}=\mu_{H}$ to (35) shows, not surprisingly, that high discounting implies a lower optimal protection of IPR.

\subsection{Other Competition Policies}

In practice, several other competition policies, besides licensing fees and intellectual property rights, are used in order to affect the profitability of standardization. We now briefly discuss the implications of such policies. Suppose that the government can directly affect markups in the hi-tech and the low-tech sectors. In particular, it can set $\epsilon_{H} \geq \epsilon$ and $\epsilon_{L} \geq \epsilon$ in the pricing equations (5).

When markups vary across firms, profits (16) become:

$$
\pi_{L}=\frac{y^{1 / \epsilon}}{\epsilon_{L}}\left(\frac{L}{1-n}\right)^{\frac{\epsilon-1}{\epsilon}} \text { and } \pi_{H}=\frac{y^{1 / \epsilon}}{\epsilon_{H}}\left(\frac{H}{n}\right)^{\frac{\epsilon-1}{\epsilon}}
$$

From $r_{L}(n)=\pi_{L} / \mu_{L}$ and the above expressions, it is immediate to see that the BGP $n$ only depends on the product $\mu_{L} \epsilon_{L}$. This result highlights that competition policy $\left(\epsilon_{L}\right)$ and IPR protection $\left(\mu_{L}\right)$ are substitutes. Intuitively, with lower mark-ups (high $\epsilon_{L}$ ) for low-tech firms, there is less entry in the $L$-sector. Yet, the government can offset this effect by reducing $\mu_{L}$, so that it becomes easier to standardize. On the contrary, $g^{s s}(n)$ does not depend on $\epsilon_{L}$, so that $n^{*}$ is as before. Given that intervening on $\mu_{L}$ or $\epsilon_{L}$ is equally effective to implement a desired $n^{*}$, the optimal mix depends on the relative costs of the two policies.

Now when we also have $\rho \rightarrow 0$, equation (35) becomes:

$$
\mu_{L}=\frac{\epsilon_{H}}{\epsilon_{L}} \cdot \frac{\mu_{H} \epsilon}{1-\epsilon+n^{*} \epsilon} .
$$

Then, under the assumptions that $\epsilon_{L}$ can be changed at no cost, it is easy to see that 
the optimal policy is:

$$
\begin{aligned}
\epsilon_{H} & =\epsilon \\
\mu_{L}^{*} & =\mu_{L}^{\min } \\
\epsilon_{L} & =\frac{\epsilon_{H}}{\mu_{L}^{\min }} \cdot \frac{\mu_{H} \epsilon}{1-\epsilon+n^{*} \epsilon}
\end{aligned}
$$

where $\mu_{L}^{\min } \geq 0$ is the minimum "technical" cost of standardization (i.e., with no IPR protection). Intuitively, full monopoly among hi-tech firms ensures high innovation; $\mu_{L}^{*}=$ $\mu_{L \text { min }}$ minimizes the resources spent on standardization; high competition among lowtech firms yields the optimal $n^{*}$. If the desired level of competition $\epsilon_{L}$ cannot be achieved, then $\mu_{L}^{*}$ should be adjusted upward accordingly. ${ }^{16}$

\subsection{North-South Trade and IPR Policy}

We now ask how trade opening in countries with a large supply of unskilled workers affects the optimal IPR policy. This question is interesting because there is an unsettled debate on whether trade liberalization in less developed countries should be accompanied by tighter IPR protection, as implied by the TRIPs Agreement, or by less strict IPR policies, which serve to encourage technology diffusion to less advanced economies. We can investigate this question using our model.

Consider an integrated world economy (the North), described by the model in Section 2. For simplicity, let us also assume that there is a single large developing country endowed with unskilled workers only (the South). Without trade, we assume that Northern technologies are copied at no cost by competitive firms in the South. However, this form of technology transfer is imperfect: when a low-tech good is introduced in the South, labor productivity there is only a fraction $\varphi \in(0,1]$. There is no innovation in the South.

Now imagine that the South opens its economy to trade. We assume that economic integration allows Northern firms to produce in the South. In the new integrated equilibrium factor prices are equalized (or else firms would relocate to the country where labor is cheaper) and Southern firms are replaced by their Northern counterpart. This result stems from the fact that Northern firms are more productive and can capture the entire market by charging a price equal to or lower than the marginal cost of the Southern

\footnotetext{
${ }^{16}$ Another way to highlight the same result is that policy does not affect markups, but rather patent duration in the low-tech sector. In the model considered so far, patent length is infinite in the lowtech sector. Suppose, however, that patent duration is finite and, once the patent expires, the good is produced by unskilled workers under competitive conditions. Here, the key trade-off is between the cost of standardization and the duration of the subsequent monopoly position in the low-tech sector. The gist of the argument is that the best combination is, in a sense, low IPR everywhere (low $\mu_{L}$ and short patents). However, it has to be carefully tailored, since the cost-relative-to-duration must be pinned down so as to get the right $n$.
} 
imitators, $p_{L} \leq w_{L} / \varphi$. However, if $\varphi>(1-1 / \epsilon)$, Northern firms must compress their markup to keep Southern imitators out.

In sum, the effect of trade opening in the South is isomorphic to an increase in the world endowment of $L$ and possibly a reduction in the markup and profit margins of low-tech firms (higher $\epsilon_{L}$ ). What are the implications for the BGP growth rate and the optimal IPR policy? The change in $L$ and $\epsilon_{L}$ have opposite effects on $\pi_{L}$ (see equation (36)) and hence on the return from standardization, so the $r_{L}(n)$ curve in Figure 1 may either shift up or down. The $r_{H}^{s s}(n)$ curve, instead, always shifts up because the greater supply of low-tech goods increases the price and thus the profitability of hi-tech products. As a result, in the new BGP, $n^{s s}$ and $g^{s s}$ may be higher or lower. Despite this ambiguity, it is easy to see that trade opening is necessarily growth (and welfare) enhancing if IPR policy, $\mu_{L}$, is correctly adjusted. This follows immediately from the upward shift of the $r_{H}^{s s}(n)$ curve, implying that the maximum attainable $r$ must be higher.

The crucial question, then, is how $\mu_{L}$ should be changed. As already seen, a higher $L / H$ increases the optimal level of IPR protection, $\mu_{L}^{*}$. On the other hand, higher competition among low-tech firms, $\epsilon_{L}$, calls for a reduction in $\mu_{L}$, to compensate for the fall in profit margins (see equation (37)). The net effect depends on which force dominates. If the liberalizing country is large and inefficient (low $\varphi$ ), the competitive pressure posed by imitators on low-tech firms is weak, while the threat to hi-tech firms, due to the increased incentives to standardize, is high. In this case, integration should be followed by a tightening of IPR policies. ${ }^{17}$

\section{Multiple Equilibria and Poverty Traps}

We have so far imposed a specific tie-breaking rule, assuming that when $w_{H}=w_{L}$ the incumbent will fight the entrance (not existing at stage (ii) of the entry-exit game). This assumption implies that standardization only takes place when it is strictly profitable and therefore, $n$ necessarily stays in the range $\left(n^{\min }, 1\right)$, since at $n=n^{\text {min }}$ we would have $w_{H}=$ $w_{L}$ and given incumbent behavior, standardization would lead to negative profits. Under this tie-breaking rule, Proposition 4 established the uniqueness of a BGP equilibrium. In this section, we show that under the alternative tie-breaking rule, whereby at $w_{H}=w_{L}$ incumbents facing the entry of a low-tech competitor exit, the model may generate multiple equilibria and potential poverty traps. ${ }^{18}$

Throughout this section, we assume a different tie-breaking rule, by which compe-

\footnotetext{
${ }^{17}$ These are the policies that a world planner would choose starting from the optimum. Yet, governments of individual countries face different incentives, because an increase in $\mu_{L}$ leads to a higher skill premium and redistributes income towards skill-abundant countries. This conflict of interests between the North and the South is studied, among others, by Grossman and Lai (2004).

${ }^{18}$ Multiple equilibria can also arise if we relax Assumption 2, while maintaining the same tie-breaking rule as in the analysis so far. However, this happens for a smaller set of parameter values. We therefore focus on relaxing the tie-breaking rule while maintaining Assumption 2.
} 


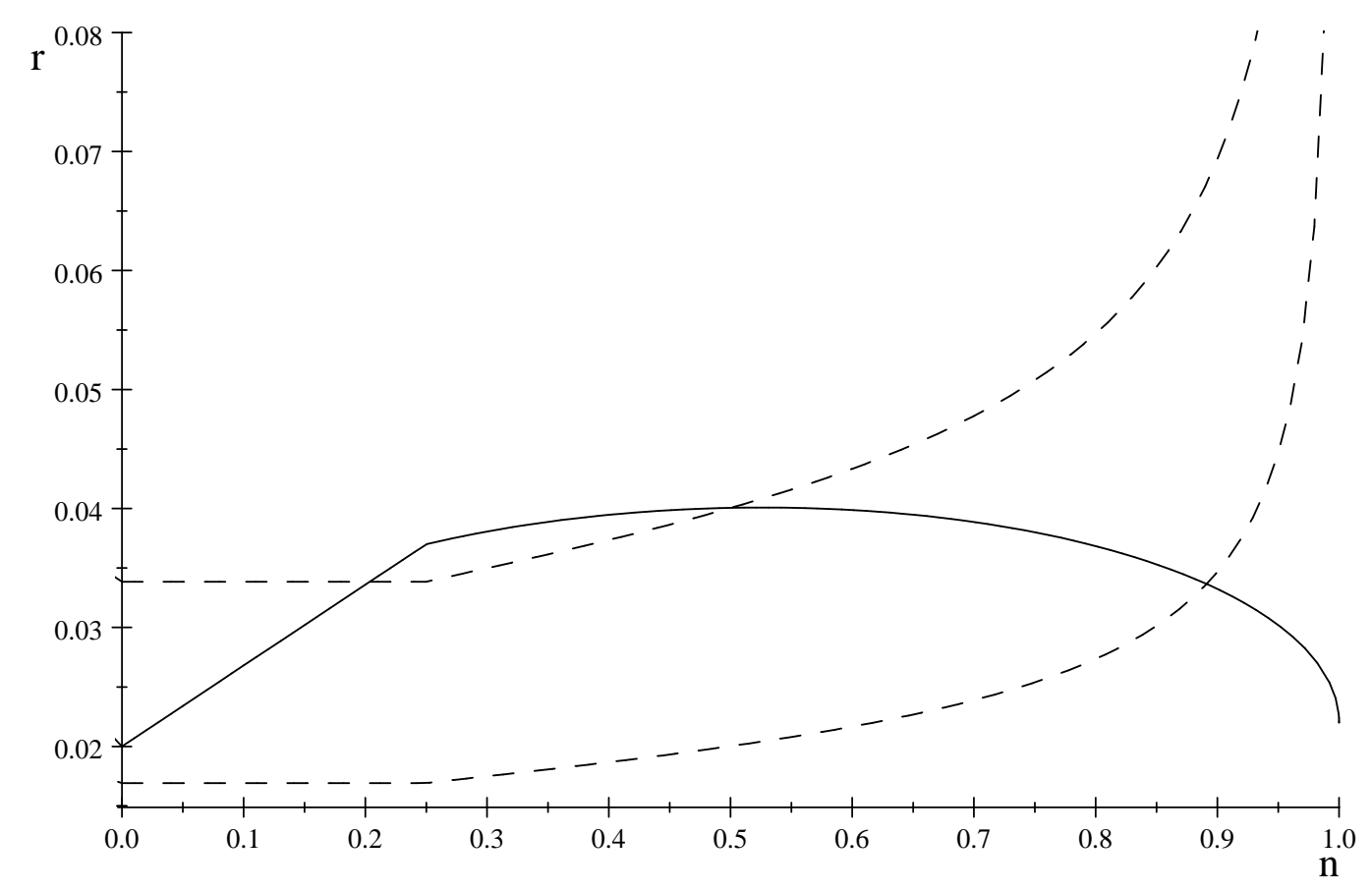

Figure 3: Solid $=r_{H}^{s s}(n)$, Dashed $=r_{L}(n)$

tition between hi-tech incumbents and entrants at $w_{H}=w_{L}$ is resolved by incumbents exiting at stage (ii) of the entry-exist game. Clearly, even under this alternative tiebreaking rule, equilibrium must involve $w_{H} \geq w_{L}$ for any $n \in[0,1]$ since skilled worker can always take unskilled jobs. But in contrast to Proposition 1 now standardization may continue even at $n \leq n^{\text {min }}$.

As a first step in the analysis of this case, we characterize the static equilibrium for low levels of $n$. Recall that $w_{H}=w_{L}$ at $n=n^{\mathrm{min}}$. For $n \leq n^{\mathrm{min}}$, the skill premium is constant at $w_{H}=w_{L}$ and some high skill workers are employed in low-tech firms. In this case, the allocation of labor between the two type of firms, $h$, is determined endogenously by equation (9) after setting $w_{H}=w_{L}$. This yields $h=n /(1-n)$ and a profit rate of $\pi_{H}=\pi_{L}=\frac{L+H}{\epsilon}$. In other words, for sufficiently low $n$, it is as if workers were perfect substitutes, prices are equalized $p_{H}=p_{L}$, and so are profits.

To find the steady states, we draw the $r_{L}(n)$ and $r_{H}^{s s}(n)$ schedules over the entire domain $n \in[0,1]$. Figure 3 shows the determination of $n^{s s}$ for two possible $r_{L}(n)$ schedules, corresponding to different values of $\mu_{L}$. Compared to Figure 1, the first part of both schedules is a straight line, as there the skill premium is constant and equal to one. The interior BGPs are again the intersections between the $r_{H}^{s s}(n)$ (solid line) and $r_{L}(n)$ (dashed line) schedules.

In addition to balanced growth equilibria, now there might exist "corner steady- 
states" such that $n=g=m=0$ and $r=\rho$. A corner steady state can arise in two different circumstances: (i) at $n=0$, there is no incentive to innovate nor to standardize, i.e., $\rho>\frac{\pi_{L}}{\mu_{L}}=\frac{H+L}{\mu_{L} \epsilon}$ and $\rho>\frac{\pi_{H}}{\mu_{H}}=\frac{H+L}{\mu_{H} \epsilon}$; (ii) at $n=0$, firms have an incentive to standardize, i.e., $\rho<\frac{H+L}{\mu_{L} \epsilon}$, but there are no goods to standardize, since $n=0$. Moreover, innovation is discouraged by the expectation that new hi-tech goods would trigger a high standardization rate. Formally, innovating firms expect that $m>\frac{H+L}{\mu_{H} \epsilon}-\rho$ whenever $n>0$. This conjecture does not violate the resource constraint since the absolute investment in standardization would be infinitesimal when $n=0$ even though the standardization rate is very high. The uninteresting case in which $r_{L}(n)$ lays above $r_{H}^{s s}(n)$ for all $n$ is still ruled out by Assumption 2 .

As shown in Figure 3, depending on the standardization cost, there are two regimes:

High $\mu_{L}$ : For $\mu_{L}>(H+L) /(\rho \epsilon)$ (lower $r_{L}(n)$ schedule in Figure 3$)$, there is a unique steady state (BGP) corresponding to the unique crossing point of the $r_{L}(n)$ and $r_{H}^{s s}(n)$ schedules.

Low $\mu_{L}$ : For $\mu_{L}<(H+L) /(\rho \epsilon)$ (upper $r_{L}(n)$ schedule) there are two interior and a corner steady state. The two interior steady states can be seen in Figure 3 . In this case, a corner steady state also exists, since $r_{H}^{s s}(0)=\rho<r_{L}(0)=(H+L) /\left(\mu_{L} \epsilon\right)$. Hence, standardization is profitable at $n=0$.

The reason for the potential multiplicity is a complementarity between investment decisions of firms. If firms expect $n$ to be high in the BGP, they also anticipate a low standardization rate, $m$, and this encourages further innovation. Greater innovation in turn increases the demand for resources (i.e., the demand for "investment" rather than consumption) and raises the interest rate. A greater interest rate reduces the value of standardization more than the value of innovation), confirming the expectation of a low $m$. In contrast, when a large fraction of the resources of the economy are devoted to standardization, expected returns from innovation decline and this limits innovative. Expectation of lower innovation reduces the interest rates, leading to reverse reasoningi.e., encouraging standardization (more than innovation) and confirming the expectation of a high $m .{ }^{19}$ Note that this complementarity was also present in the model analyzed in the previous sections, but, it did not give rise to multiplicity because Assumption 2 guaranteed that the other candidate steady states involved levels of $n$ below $n^{\text {min }}$, which

\footnotetext{
${ }^{19}$ To see the role of the interest rate in this reasoning, consider a more general formulation of preferences where $\theta$ is the inverse of the intertemporal elasticity of substitution. In this case, the Euler equation takes the form $\dot{C} / C=(r-\rho) / \theta$. Using this, equation (26) becomes:

$$
r_{H}^{s s}(n)=\frac{\theta n}{n \theta+1-n} \frac{\pi_{H}}{\mu_{H}}+\rho\left(\frac{1-n}{n \theta+1-n}\right) .
$$
}

Note that, as $\theta \rightarrow 0$ the $r_{H}^{s s}(n)$ curve becomes flat and the BGP is necessarily unique. 
were ruled out by Proposition 1 under our baseline tie-breaking rule. Thus, the fact that standardization was profitable only when unskilled labor was strictly cheaper than skilled labor prevented the economy from falling to low-growth traps where innovation is discouraged by the expectation of a very fast standardization rate.

We summarize the characterization of the set of steady-state equilibria in the following proposition (proof in the text).

Proposition 9 Suppose that Assumptions 1 and 2 hold and adopt the tie-breaking rule that when $w_{H}=w_{L}$, incumbents exit in state (ii) of the sequential entry-exit game. Then:

1. If $\mu_{L}>(H+L) /(\rho \epsilon)$, there exists a unique BGP which is interior.

2. If $\mu_{L}<(H+L) /(\rho \epsilon)$, there exist two interior BGP equilibria and a corner steady state.

It is also noteworthy that the non-monotonic relationship between the $g^{s s}$ and $\mu_{L}$ and the policy analysis derived in the previous sections now apply to the higher interior BGP. The main novelty, however, is that too low a cost of standardization may lead to multiple steady states, one of them in fact corresponding to stagnation, and the exact equilibrium path is determined by self-fulfilling expectations.

\section{Concluding Remarks}

New technologies often diffuse as a result of costly adoption and standardization decisions. Such standardization also creates cheaper ways of producing new products, for example, substituting cheaper unskilled labor for the more expensive skilled labor necessary for the production of new complex products. This process endogenously generates competition to original innovators. In this paper, we studied the implications of this costly process of standardization, emphasizing both its role as an engine of growth and its potential negative effects on innovation (because of the "business stealing" effect that it creates).

Our analysis has delivered a number of new results. First, the tension between innovation and standardization generates an inverse U-shaped relationship between competition and growth. Second, while technology diffusion is potentially beneficial, it can also have destabilizing effects. Standardization can open the door to multiple equilibria (multiple growth paths). Finally, we characterized the optimal competition and IPR policy and how it depends on endowments and other parameters, such as the elasticity of substitution between products. We found that innovation rents should be protected more when skilled workers are perceived as scarcer, that is, when they are in short supply and when the elasticity of substitutions between goods is high. We also showed that 
these results provide new reasons for linking North-South trade to intellectual property rights protection.

It is also worth noting that a key feature of our analysis is the potential competition between standardized products and the original hi-tech products. We believe that this is a good approximation to a large number of cases in which standardization takes place by different firms (and often in the form of slightly different products). Nevertheless, the alternative, in which standardization is carried out by the original innovator, is another relevant benchmark. In our follow-up work, Acemoglu, Gancia and Zilibotti (2010), we study a model of offshoring, where offshoring can be viewed as a costly process of standardization carried out by the original innovator to make goods producible in less developed countries with cheaper labor.

Our model yields a number of novel predictions that can be investigated empirically. In particular, it suggests that competition and IPR policy should have an impact on skill premia. Furthermore, data on product and process innovation might be used to test the existence of a trade-off between innovation and standardization at the industry level. These seem interesting directions for future work.

\section{Appendix}

\subsection{Proof of Lemma 1}

Recall $\pi_{H}=\frac{p_{H} H}{\epsilon_{H} A_{H}}=\frac{p_{H} H}{\epsilon_{H} n A}$. From (11) it is immediate to see that $\frac{\partial \pi_{H}}{\partial n}<0$ if $p_{H} \geq p_{L}$, which is true in equilibrium. To establish the properties of $\pi_{L}$, note that:

$$
\frac{\partial(\epsilon-1) \ln \pi_{L}}{\partial n}=\frac{\frac{1}{\epsilon}\left(\frac{n}{1-n}\right)^{\frac{\epsilon+1}{\epsilon}}\left(\frac{1}{n^{2}}\right) h^{\frac{\epsilon-1}{\epsilon}}}{1+\left(\frac{n}{1-n}\right)^{\frac{1}{\epsilon}} h^{\frac{\epsilon-1}{\epsilon}}}+\frac{\epsilon-2}{1-n}>0 \text { if } \epsilon>2-\frac{\frac{1}{\epsilon} \frac{1}{n} h^{\frac{\epsilon-1}{\epsilon}}}{\left(\frac{1-n}{n}\right)^{\frac{1}{\epsilon}}+h^{\frac{\epsilon-1}{\epsilon}}}
$$

For $\epsilon \geq 2, \lim _{n \rightarrow 1} \frac{\partial \pi_{L}}{\partial n}=\infty$. Convexity of $\pi_{L}$ follows immediately because the function $\frac{\partial \pi_{L}}{\partial n}$ has no critical point.

\subsection{Notes on Figures}

The benchmark economy used to draw all figures has the following parameter values:

$$
\rho=0.02 ; \epsilon=2 ; \mu_{H}=22.7, \mu_{L}=59.1 ; H=1 ; L=3
$$

implying in steady state:

$$
g=0.02 ; r=0.02 ; m=0.02 ; n=0.5 ; \frac{w_{H}}{w_{L}}=1.5 .
$$




\subsection{Proof of Proposition 4}

A BGP must be a rest point of the dynamical system (21)-(22). We first note that there cannot be a rest point at the boundaries $n=n^{\text {min }}$ and $n=1$ in view of Proposition 1 . Thus any BGP must be interior as defined in Proposition 3, or equivalently, it must be a zero of the dynamical system (21)-(22). We denote such a zero by $\left(n^{s s}, \chi^{s s}\right)$, where $n^{s s}$ satisfies $r_{L}\left(n^{s s}\right)=r_{H}^{s s}\left(n^{s s}\right)$ (see again Proposition 3 ). We prove the existence of a unique interior BGP by showing that there is a unique value $n^{s s} \in\left(n^{\text {min }}, 1\right)$ such that $r_{L}\left(n^{s s}\right)=r_{H}^{s s}\left(n^{s s}\right)$, that there is a unique corresponding value of $\chi^{s s}$ and that at $\left(n^{s s}, \chi^{s s}\right)$ the transversality condition is satisfied.

We prove the first step by establishing that $r_{H}^{s s}\left(n^{s s}\right)$ is a continuous inverse U-shaped function whereas $r_{L}\left(n^{s s}\right)$ is a continuous, increasing and convex function. Moreover $r_{H}^{s s}\left(n^{\min }\right)>r_{L}\left(n^{\mathrm{min}}\right)$ (from Assumption 2) and $\lim _{n \rightarrow 1}\left(r_{L}\left(n^{s s}\right)-r_{H}^{s s}\left(n^{s s}\right)\right)=\infty$. Then, the intermediate value theorem establishes the existence of such a BGP, while the shape of the two functions implies uniqueness. Let $\phi(x) \equiv\left[x+x\left(\frac{1}{x}-1\right)^{\frac{1}{\epsilon}} h^{\frac{1-\epsilon}{\epsilon}}\right]^{\frac{1}{\epsilon-1}}$ where $\epsilon \geq 2$ and $h \geq 0$. Standard algebra establishes that $\phi(x)$ is a continuous inverse Ushaped concave function, such that $\lim _{x \rightarrow 0} \phi^{\prime}(x)=\infty$ and $\lim _{x \rightarrow 1^{-}} \phi^{\prime}(x)=-\infty$. Thus, $\phi(x)$ has a unique interior maximum in the unit interval. Next, note that $r_{H}^{s s}\left(n^{s s}\right)=$ $\phi\left(n^{s s}\right) \cdot H /\left(\mu_{H} \epsilon\right)+\left(1-n^{s s}\right) \rho$. Since $r_{H}^{s s}\left(n^{s s}\right)$ is a linear transformation of $\phi\left(n^{s s}\right)$, it is also a continuous inverse U-shaped concave function, with a unique interior maximum in the unit interval. Consider now $r_{L}\left(n^{s s}\right)$. Since $r_{L}\left(n^{s s}\right)=\pi_{L}\left(n^{s s}\right) / \mu_{L}$, Lemma 1 establishes that $r_{L}\left(n^{s s}\right)$ is increasing and convex, with $\lim _{n^{s s} \rightarrow 1} r_{L}\left(n^{s s}\right)=\infty$ (thus, $\left.\left.\lim _{n^{s s} \rightarrow 1}\left[r_{L}\left(n^{s s}\right)-r_{H}^{s s}\left(n^{s s}\right)\right]=\infty\right)\right)$.

Next, straightforward algebra immediately implies that, conditional on $n=n^{s s}$, $m=m\left(n^{s s}\right)$ and $z=z\left(n^{s s}\right)$ there exists a unique value $\chi=\chi^{s s}$ that yields a zero of the dynamical system (21)-(22). Finally, since in BGP $r=\rho+g>g$ (from (1) and Assumptions 1-2), the transversality condition is satisfied in the unique candidate BGP.

\subsection{Proof of Proposition 5}

Recall that dynamic equilibria are given by solutions to dynamical system (21)-(22) with boundary conditions given by the initial condition $n=n_{0}$ and the transversality condition. By the same argument as in the proof of Proposition 4, there cannot be any dynamic equilibrium path where $n \rightarrow n^{\text {min }}$ and $n \rightarrow 1$. Any dynamic equilibrium must thus either converge to the unique (interior) BGP $\left(n^{s s}, \chi^{s s}\right)$ or involves cycles. We will show in this proof that starting from any initial condition $n=n_{0}$ in the neighborhood of $n^{s s}$ (the BGP), there exists a unique path converging to $\left(n^{s s}, \chi^{s s}\right)$ and that there cannot be cycles, thus establishing local saddle-path stability of the dynamic equilibrium.

Because there are two sources of technical change (innovation and standardization), we first distinguish between three possible types of potential dynamic equilibria (which may converge to the BGP, $\left.\left(n^{s s}, \chi^{s s}\right)\right)$. 
CASE 1: $V_{H}=\mu_{H}$ and $V_{L}<\mu_{L}\left(\Rightarrow m=0\right.$ and $\left.g=(y(n)-\chi) / \mu_{H}\right)$. In this case, from Proposition 3 the dynamics are governed by the following system of ordinary differential equations:

$$
\begin{aligned}
\frac{\dot{\chi}}{\chi} & =\frac{\pi_{H}(n)}{\mu_{H}}-\rho-\frac{y(n)-\chi}{\mu_{H}} \\
\dot{n} & =(1-n) \frac{y(n)-\chi}{\mu_{H}} .
\end{aligned}
$$

CASE 2: $V_{H}<\mu_{H}$ and $V_{L}=\mu_{L}\left(\Rightarrow m=(y(n)-\chi) /\left(n \mu_{L}\right)\right.$ and $\left.g=0\right)$. In this case, again from Proposition 3 the dynamics are governed by the following system of ordinary differential equations:

$$
\begin{aligned}
\frac{\dot{\chi}}{\chi} & =\frac{\pi_{L}(n)}{\mu_{L}}-\rho \\
\dot{n} & =-\left(\frac{y(n)-\chi}{\mu_{L}}\right) .
\end{aligned}
$$

CASE 3: $V_{H}=\mu_{H}$ and $V_{L}=\mu_{L}\left(\Rightarrow m=\pi_{H}(n) / \mu_{H}-\pi_{L}(n) / \mu_{L}\right.$ and $g=$ $\left.\left(y(n)-\mu_{L} m n-\chi\right) / \mu_{H}\right)$. In this case, the dynamics are governed by the following system of ordinary differential equations:

$$
\begin{aligned}
\frac{\dot{\chi}}{\chi} & =\frac{\pi_{L}(n)}{\mu_{L}}-\rho-\frac{y(n)-\mu_{L} m n-\chi}{\mu_{H}} \\
\dot{n} & =(1-n) \frac{y(n)-\chi}{\mu_{H}}-m n\left(1+(1-n) \frac{\mu_{L}}{\mu_{H}}\right) .
\end{aligned}
$$

In all three cases, the differential equations are defined over the region $\chi \in[0, y(n)]$, $n \in\left[n_{\min }, 1\right]$. Recall that in the BGP $m>0$ and $g>0$. This implies that $V_{H}=\mu_{H}$ and $V_{L}=\mu_{L}$. Therefore, $\left(n^{s s}, \chi^{s s}\right)$ is a zero of the dynamical system (40), but it is not a zero either of (38) or of (39). Nevertheless, we will show that CASE 3 cannot describe dynamic equilibrium behavior at any point with $(n, \chi) \neq\left(n^{s s}, \chi^{s s}\right)$. Instead, the equilibrium dynamics will be given by either CASE 1 or CASE 2 (depending on whether $n$ is above or below $n^{s s}$ ) and will be unique. Then under the equilibrium dynamics, the economy will converge in finite time to $\left(n^{s s}, \chi^{s s}\right)$, and then a jump in $m$ and $r$ will create a switch to CASE 3 at that point. Since $\left(n^{s s}, \chi^{s s}\right)$ is a zero of $(40)$, the economy will have reached the BGP and will stay at $\left(n^{s s}, \chi^{s s}\right)$ thereafter.

We prove by first establishing several Lemmas. First, Lemma 2 establishes that, if $n<n^{s s}$, there exists a unique trajectory converging to $\left(n^{s s}, \chi^{s s}\right)$ (it is immediate that, if $n<n^{s s}$, there exists no trajectory converging to $\left(n^{s s}, \chi^{s s}\right)$ following the dynamics

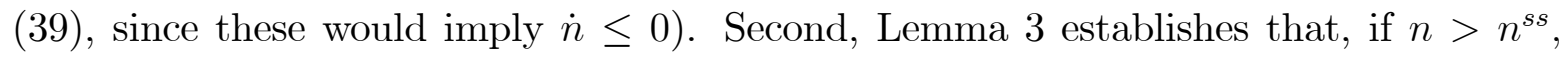
there exists a unique trajectory converging to $\left(n^{s s}, \chi^{s s}\right)$ following the dynamics (39) (it 
is immediate that, if $n>n^{s s}$, there exists no trajectory converging to $\left(n^{s s}, \chi^{s s}\right)$ following the dynamics given by (38), since these imply $\dot{n} \geq 0$ ). Third, Lemma 4 provides a complete characterization of equilibrium dynamics when the transition involves either only innovation or only standardization, followed by a jump in either the innovation of standardization rate as the economy reaches $\left(n^{s s}, \chi^{s s}\right)$, but continuous changes in asset values. Fourth, Lemma 5 establishes that (under the sufficient conditions of the Proposition) there exists no trajectory converging to $\left(n^{s s}, \chi^{s s}\right)$ following the dynamics (40). Finally, Lemma 6 rules out transitional dynamics in the neighborhood of the BGP in which there is a jump from CASE 3 to either CASE 1 or CASE 2 or between CASE 1 and CASE 2. These lemmas together establish local saddle-path stability.

Lemma 2 Suppose $n_{0}<n^{s s}$. Then, there exists a unique trajectory attaining $\left(n^{s s}, \chi^{s s}\right)$ in finite time following the dynamics of CASE 1, (38). This trajectory features monotonic convergence in $n(\dot{n}>0)$.

Proof. Consider the system of differential equations (38). This system has no zero over the feasible region $\left[n_{\min }, 1\right] \times[0, y(n)]$. In particular, in the interior of the region $\left[n_{\min }, 1\right] \times[0, y(n)]$, and hence at $\left(n^{s s}, \chi^{s s}\right), \dot{n}>0$ and $\dot{\chi} \gtreqless 0 \Leftrightarrow \chi \gtreqless \hat{\chi}(n)$, where $\hat{\chi}(n)=$ $\mu_{H} \rho+y(n)-\pi_{H}(n)<y(n)$. The last inequality follows from Assumption 1. Although whether $\hat{\chi}\left(n^{s s}\right) \gtreqless \chi\left(n^{s s}\right)$ is in general ambiguous, there is a unique trajectory (and a unique initial level of the control variable, $\left.\chi_{0}\right)$ converging in finite time to $\left(n^{s s}, \chi^{s s}\right)$. In particular, since $\left(n^{s s}, \chi^{s s}\right)$ is not a zero of the system (38), the determination of the converging trajectory can be expressed as an initial value problem with $\left(n_{T}^{s s}, \chi_{T}^{s s}\right)$ being the boundary (terminal) condition. From the standard result of existence and uniqueness of solutions for systems of ordinary differential equations, this initial value problem has a unique solution. Fixing the initial condition $n_{0}$ yields a unique solution for $T$ (the length of the transition) and $\chi_{0}$. The monotonicity of the dynamics of $n$ ensures that this solution is unique, i.e., there does not exist two solutions $\left(n_{0}, T, \chi_{0}\right)$ and $\left(n_{0}, T^{\prime}, \chi_{0}^{\prime}\right)$ with $T \neq T^{\prime}$ and $\chi_{0} \neq \chi_{0}^{\prime}$. This argument also proves that convergence is attained in finite time.

Lemma 3 Suppose $n_{0}>n^{s s}$. Then, there exists a unique trajectory attaining $\left(n^{s s}, \chi^{s s}\right)$ in finite time following the dynamics of CASE 2, (39). This trajectory features monotonic convergence $(\dot{n}<0$ and $\dot{\chi}>0)$.

Proof. The proof is similar to that of Lemma 2. The dynamical system again has no zero over the feasible region $\left[n_{\min }, 1\right] \times[0, y(n)]$. In particular, for $n_{0} \geq n^{s s}, \dot{n}>0$ and $\dot{\chi}>0$. The latter follows from the observation that $\dot{\chi} \gtreqless 0 \Leftrightarrow \pi_{L}(n) / \mu_{L} \gtreqless \rho$, where $\pi_{L}^{\prime}(n)<0$ and $\pi_{L}\left(n^{s s}\right) / \mu_{L}>\rho$, implying that $\pi_{L}(n) / \mu_{L}>\rho$ for all $n \geq n^{s s}$. Standard properties of systems of ordinary differential equations establish that there is a unique trajectory converging in finite time to $\left(n^{s s}, \chi^{s s}\right)$ and this trajectory features monotonic dynamics. 
The previous two Lemmas together imply our key characterization result.

Lemma 4 There exists equilibrium dynamics with the following characteristics. If $n_{0}<n^{s s}$, the economy converges in finite time to $\left(n^{s s}, \chi^{s s}\right)$ following the system of differential equations (38), with monotonic convergence in $n$. Throughout this convergence, $V_{H}=\mu_{H}, V_{L}<\mu_{L}, m=0$ and $g=(y(n)-\chi) / \mu_{H}$. When the economy reaches $\left(n^{s s}, \chi^{s s}\right)$, there is a discrete increase in standardization offset by a fall in the interest rate such that $r\left(n^{s s}\right)=r^{\prime}\left(n^{s s}\right)+m\left(n^{s s}\right)$. Thereafter, $V_{H}=\mu_{H}$ and $V_{L}=\mu_{L}$.

If $n_{0}>n^{s s}$ the economy converges in finite time to $\left(n^{s s}, \chi^{s s}\right)$ following the system of differential equations (39), with monotonic convergence in $n$ and $\chi$. Throughout this convergence, $V_{H}<\mu_{H}, V_{L}=\mu_{L}, m=(y(n)-\chi) /\left(n \mu_{L}\right)>0$ and $g=0$. When the economy reaches $\left(n^{s s}, \chi^{s s}\right)$, there is a discrete fall in standardization offset by an increase in innovation such that $y\left(n^{s s}\right)-\chi$ remains constant. Thereafter, $V_{H}=\mu_{H}$ and $V_{L}=\mu_{L}$

Proof. The proof follows from Lemmas 2 and 3 combined with the following observations. Suppose we start with $n_{0}<n^{s s}$, then the dynamic equilibrium is given by the system of differential equations (38), so from Lemma 2 until $T$, we have $m=0$. At $T$, we reach $\left(n^{s s}, \chi^{s s}\right)$ and $m$ jumps from zero to its steady state, $m^{s s}$. This is offset by an equal jump down in $r$ implying that $V_{H}$ does not change (i.e., it remains at $V_{H}=\mu_{H}$ ). Moreover, at $T, V_{L}$ attains its steady state (BGP) value, $V_{L}=\mu_{L}$. Note that there is no discontinuity in the asset value $V_{L}$, since the change in $r$ and $m$ are perfectly anticipated, causing a continuous change in the value $V_{L}$ before the actual change occurs to reach $V_{L}=\mu_{L}$ exactly at $T$ (the continuity of $V_{L}$ ensures that there is no arbitrage opportunity in buying and selling shares of $L$-sector firms). Thus at this point, the dynamics switch to those given by the system of differential equations (40) with both innovation and standardization. Since $\left(n^{s s}, \chi^{s s}\right)$ is a zero of $(40)$, the economy stays at $\left(n^{s s}, \chi^{s s}\right)$ thereafter. The fact that this path satisfies the transversality condition follows by the same argument as in the proof of Proposition 4.

Next, suppose that we start with $n_{0}>n^{s s}$. Then the dynamic equilibrium is given by the system of differential equations (39) and from Lemma 3, until $T, g=0$. At $T$, investment in standardization and $m_{T}$ fall discretely (the latter declining to $m^{s s}$ ), and investment in innovation and $g$ jumps up (the latter increasing to $g^{s s}$ ). There is no change in overall investment and thus neither $r$, nor consumption nor $V_{L}$ change at $T$. As $m$ jumps down, $V_{H}$ attains its stead state value, $V_{H}=\mu_{H}$ exactly at $T$ (note that the path of $V_{H}$ is continuous at $T$, as the change in $m$ is perfectly anticipated by investors). As a result, again at $T$, the dynamics switch to those given by the system of differential equations (40) with both innovation and standardization. Since $\left(n^{s s}, \chi^{s s}\right)$ is a zero of (40), the economy stays at $\left(n^{s s}, \chi^{s s}\right)$ thereafter. The fact that this path satisfies the transversality condition again follows by the same argument.. 
We next show that transitional dynamics converging to $\left(n^{s s}, \chi^{s s}\right)$ cannot feature both $V_{H}=\mu_{H}$ and $V_{L}=\mu_{L}$ since the system (40) is unstable in the neighborhood of $\left(n^{s s}, \chi^{s s}\right)$.

Lemma 5 Suppose $n_{0} \neq n^{s s}$. Then, under the (sufficient) conditions of the Proposition, there exists no trajectory converging to $\left(n^{s s}, \chi^{s s}\right)$ following the dynamical system (40).

Proof. The proof of this lemma, which is long, is presented in Appendix B, which is available online.

Lemmas 2-5 establish that in the neighborhood of $\left(n^{s s}, \chi^{s s}\right)$, we must have either $V_{H}<\mu_{H}$ or $V_{L}<\mu_{L}$, implying that there is either only innovation or only standardization. However, the results established so far do not rule out "switches" between different regimes while $n_{t} \neq n^{s s}$, and thus cycles. Moreover, with such switches, the equilibrium might also be indeterminate, with multiple paths starting from some initial $n_{0}$ converging to the BGP. Lemma 6 rules out all of these possibilities by showing that in the neighborhood of the BGP, there cannot be a switch from the dynamics given by any one of (38), (39) and (40) to one of the other two. (For notational convenience, in this lemma, we write $V_{H, t}=\mu_{H}$ to mean that $V_{H, t^{\prime}}=\mu_{H}$ for $t^{\prime}$ in a neighborhood of $t$ ).

Lemma 6 Consider an equilibrium trajectory in the neighborhood of the $B G P,\left(n^{s s}, \chi^{s s}\right)$. Then, there cannot be a switch from any one of (38), (39) and (40) to one of the other two, i.e., if at $t_{0}$ in the neighborhood of $\left(n^{s s}, \chi^{s s}\right)$, we have $V_{H, t_{0}}=\mu_{H}$ and $V_{L, t_{0}}=\mu_{L}$, then an equilibrium cannot involve $V_{H, t}<\mu_{H}$ and/or $V_{L, t}<\mu_{L}$ for $t>t_{0}$; if we have $V_{H, t_{0}}=\mu_{H}$ and $V_{L, t_{0}}<\mu_{L}$, then an equilibrium cannot involve $V_{H, t}<\mu_{H}$ and/or $V_{L, t}=\mu_{L}$ for $t>t_{0}$; and if we have $V_{H, t_{0}}<\mu_{H}$ and $V_{L, t_{0}}=\mu_{L}$, then an equilibrium cannot involve $V_{H, t}=\mu_{H}$ and $/$ or $V_{L, t}<\mu_{L}$ for $t>t_{0}$.

Proof. We will prove that if in the neighborhood of $\left(n^{s s}, \chi^{s s}\right)$, we have $V_{H, t_{0}}=\mu_{H}$ and $V_{L, t_{0}}=\mu_{L}$, then an equilibrium cannot involve $V_{H, t}=\mu_{H}$ and $V_{L, t}<\mu_{L}$ for $t>t_{0}$. The other cases are analogous.

Suppose to obtain a contradiction that this is the case and denote the last instance where $V_{L}=\mu_{L}$ by $T$ (i.e., $V_{L, T+\varepsilon}<\mu_{L}$ for $\varepsilon>0$. We need to distinguish two cases. First, $V_{L, t^{\prime}}<\mu_{L}$ for all $t^{\prime}>t$, and second, there exists $T^{\prime}>t$, such that we again have $V_{L, T^{\prime}}=\mu_{L}$.

Case 1: the fact that $V_{L, t^{\prime}}<\mu_{L}$ for all $t^{\prime}>t$ contradicts the hypothesis that the equilibrium path will converge to the BGP.

Case 2: we write $V_{L, T}$ as follows:

$$
\begin{aligned}
V_{L, T} & =\int_{T}^{\infty} \exp \left(-\int_{T}^{\tau} r\left(n_{\nu}\right) d \nu\right) \pi_{L}\left(n_{\tau}\right) d \tau \\
& =\int_{T}^{T^{\prime}} \exp \left(-\int_{T}^{\tau} r\left(n_{\nu}\right) d \nu\right) \pi_{L}\left(n_{\tau}\right) d \tau+\exp \left(-\int_{T}^{T^{\prime}} r\left(n_{\tau}\right) d \tau\right) \mu_{L}
\end{aligned}
$$


where the equality exploits the fact that by hypothesis $V_{L, T^{\prime}}=\mu_{L}$. Moreover, we also have, again by hypothesis, that $V_{L, T}=\mu_{L}$, which implies

$$
\int_{T}^{T^{\prime}} \exp \left(-\int_{T}^{\tau} r\left(n_{\nu}\right) d \nu\right) \pi_{L}\left(n_{\tau}\right) d \tau=\left(1-\exp \left(-\int_{T}^{T^{\prime}} r\left(n_{\tau}\right) d \tau\right)\right) \mu_{L}
$$

Suppose next that $n_{T}>n^{s s}$. By the instability result in Lemma 5 , this implies $\dot{n}_{T}>0$ and thus $n_{\tau}>n^{s s}$ for all $\tau \in\left[T, T^{\prime}\right]$. But then from Lemma $1, \pi_{L}\left(n_{\tau}\right)>\pi_{L}\left(n^{s s}\right)$ for all $\tau \in\left[T, T^{\prime}\right]$. Moreover, since $V_{H, \tau}=\mu_{H}$ and $V_{L, \tau}<\mu_{L}$, we also have that for all $\tau \in\left[T, T^{\prime}\right]$

$$
r\left(n_{\tau}\right)=\frac{\pi_{H}\left(n_{\tau}\right)}{\mu_{H}}>\frac{\pi_{L}\left(n_{\tau}\right)}{\mu_{L}}>\frac{\pi_{L}\left(n^{s s}\right)}{\mu_{L}}=r\left(n^{s s}\right),
$$

where the second inequality again follows from Lemma 1 in view of the fact that $n_{\tau}>n^{s s}$ for all $\tau \in\left[T, T^{\prime}\right]$. But then,

$$
\begin{aligned}
\int_{T}^{T^{\prime}} \exp \left(-\int_{T}^{\tau} r\left(n_{\nu}\right) d \nu\right) \pi_{L}\left(n_{\tau}\right) d \tau & <\left(\int_{T}^{T^{\prime}} \exp \left(-\int_{T}^{\tau} r\left(n_{\nu}\right) d \nu\right) d \tau\right) \pi_{L}\left(n_{s s}\right) \\
& <\left(1-\exp \left(-\int_{T}^{T^{\prime}} r\left(n_{\tau}\right) d \tau\right)\right) \mu_{L}
\end{aligned}
$$

where the second inequality follows from the fact that $r\left(n_{\tau}\right)<r\left(n^{s s}\right)$ for all $\tau \in\left[T, T^{\prime}\right]$. This inequality contradicts (41).

Suppose instead that $n_{T}<n^{s s}$. By the instability result in Lemma $5, \dot{n}_{T}<0$ and thus $n_{\tau}<n^{s s}$ for all $\tau \in\left[T, T^{\prime}\right]$. Moreover, by the same reasoning for all $\tau \in\left[T, T^{\prime}\right]$, $\pi_{H}\left(n_{\tau}\right)>\pi_{H}\left(n^{s s}\right)$ and since $V_{L, \tau}<\mu_{L}, m\left(n_{\tau}\right)=0$. Therefore,

$$
\begin{aligned}
V_{H, T} & =\int_{T}^{\infty} \exp \left(-\int_{T}^{\tau}\left(r\left(n_{\nu}\right)+m\left(n_{\nu}\right)\right) d \nu\right) \pi_{H}\left(n_{\tau}\right) d \tau \\
& =\int_{T}^{T^{\prime}} \exp \left(-\int_{T}^{s} r\left(n_{\nu}\right) d \nu\right) \pi_{H}\left(n_{\tau}\right) d \tau+\exp \left(-\int_{T}^{T^{\prime}} r\left(n_{\tau}\right) d \tau\right) \mu_{H}
\end{aligned}
$$

But since for all $\tau \in\left[T, T^{\prime}\right]$

$$
r\left(n_{\tau}\right)=\frac{\pi_{H}\left(n_{\tau}\right)}{\mu_{H}}>\frac{\pi_{H}\left(n^{s s}\right)}{\mu_{H}}
$$

the first term in (42) is strictly greater than $\left(1-\exp \left(-\int_{t}^{T} r\left(n_{\tau}\right) d \tau\right)\right) \mu_{H}$ and thus contradicts $V_{H, T}=\mu_{H}$.

Lemmas 2-6 establish the results of the Proposition. In particular, Lemmas 5 and 6 imply that starting at $n_{t} \neq n^{s s}$ in the neighborhood of the BGP we must have $V_{H}<\mu_{H}$ 
or $V_{L}<\mu_{L}$. If $V_{H}=\mu_{H}$ and $V_{L}=\mu_{L}$, either we diverge from the BGP in view of 5 , or we have to switch to a regime where $V_{H}<\mu_{H}$ or $V_{L}<\mu_{L}$, which is ruled out by Lemma 6. If we have $V_{H}<\mu_{H}$ or $V_{L}<\mu_{L}$ in the neighborhood of the BGP, then Lemmas 2-4 imply that there exists a unique path converging to the BGP. This completes the proof of the Proposition.

\section{REFERENCES}

[1] Acemoglu, Daron (1998), "Why Do New Technologies Complement Skills? Directed Technical Change and Wage Inequality" Quarterly Journal of Economics 113, 10551090.

[2] Acemoglu, Daron (2002), "Directed Technical Change," Review of Economic Studies, 69, 781-809.

[3] Acemoglu Daron (2003), "Patterns of Skill Premia," Review of Economic Studies, $70,199-230$.

[4] Acemoglu, Daron (2005). "Modeling Inefficient Economic Institutions," in Advances in Economic Thoery, Proceedings of 2005 World Congress.

[5] Acemoglu, Daron (2009), Introduction to Modern Economic Growth, Princeton University Press.

[6] Acemoglu, Daron, Philippe Aghion and Fabrizio Zilibotti (2006), "Distance to Frontier, Selection and Economic Growth," Journal of the European Economic Association $4,37-74$.

[7] Acemoglu, Daron, Gino Gancia and Fabrizio Zilibotti (2010), "Offshoring, Innovation and Wages," mimeo.

[8] Acemoglu, Daron and Fabrizio Zilibotti (2001), "Productivity Differences," Quarterly Journal of Economics 116, 563-606.

[9] Aghion Philippe, Nick Bloom, Richard Blundell, Rachel Griffith and Peter Howitt, (2005). "Competition and innovation: an inverted-U relationship," Quartertly Journal of Economics 120(2): 701-28.

[10] Aghion, Philippe, Christopher Harris, Peter Howitt and John Vickers (2001), "Competition, Imitation, and Growth with Step-by-Step Innovation," Review of Economic Studies, 68, 467-492.

[11] Aghion, Philippe and Peter Howitt (1992), "A Model of Growth through Creative Destruction," Econometrica, 60, 2, 323-351. 
[12] Aghion, Philippe, Peter Howitt and Gianluca Violante (2002). "General Purpose Technology and Wage Inequality," Journal of Economic Growth 7, 315-345.

[13] Antras, Pol (2005). "Incomplete Contracts and the Product Cycle," American Economic Review, 95, 1054-1073.

[14] Atkinson, Anthony and Joseph Stiglitz (1969). "A New View of Technological Change," Economic Journal, 573-578.

[15] Autor, David, Frank Levy and Richard Murnane (2003). "The Skill Content of Recent Technological Change: An Empirical Exploration." Quarterly Journal of Economics, 118, 1279-1334.

[16] Basu, Susanto and David Weil (1998), "Appropriate Technology and Growth," Quarterly Journal of Economics 113, 1025-1054.

[17] Bessen, James and Eric Maskin (2009). "Sequential Innovation, Patents, and Innovation," RAND Journal of Economics, 40, 611-63.

[18] Bhattacharya, Sudipto and Sergei Guriev (2006). "Patents vs. Trade Secrets: Knowledge Licensing and Spillover" Journal of the European Economic Association, 4, 1112-1147.

[19] Boldrin Michele and David Levine, (2005). "IP and Market Size," mimeo.

[20] Bonfiglioli, Alessandra and Gino Gancia (2008). "North-South Trade and Directed Technical Change," Journal of International Economics, 76, 276-296.

[21] Broda, Christian and David E. Weinstein (2006). "Globalization and the Gains from Variety," Quarterly Journal of Economics, 121, 541-585.

[22] Caselli, Francesco (1999). "Technological revolutions," American Economic Review $89,78-102$.

[23] Chari, V. V., Mikhail Golosov and Aleh Tsyvinski (2009). "Prizes and Patents: Using Market Signals to Provide Incentives for Innovations" working paper.

[24] Chari V.V. and Hugo Hopenhayn (1991). "Vintage Human Capital, Growth, and the Diffusion of New Technology," Journal of Political Economy, 99, 1142-1165.

[25] Cohen, Wesley M. and Steven Klepper (1996). "Firm Size and the Nature of Innovation within Industries: The Case of Process and Product R\&D," The Review of Economics and Statistics, 78, 232-243. 
[26] David, Paul (1975) Technical Change, Innovation and Economic Growth: Essays on American and British Experience in the Nineteenth Century, London: Cambridge University Press.

[27] Dinopoulos, Elias and Paul Segerstrom (2007). "North-South Trade and Economic Growth," Stockholm School of Economics, mimeo.

[28] Dinopoulos, Elias and Paul Segerstrom (2009). "Intellectual Property Rights, Multinational Firms and Economic Growth," Journal of Development Economics, forthcoming.

[29] Galor, Oded and Omar Moav (2000) "Ability-Biased Technological Transition, Wage Inequality, and Economic Growth," Quarterly Journal of Economics 115, 469-497.

[30] Galor, Oded and Daniel Tsiddon (1997) "Technological Progress, Mobility, and Economic Growth," The American Economic Review, 87, 363-382.

[31] Gancia, Gino, Andreas Müller and Fabrizio Zilibotti (2010). "Structural Development Accounting," University of Zurich and CREI, mimeo.

[32] Gancia, Gino and Fabrizio Zilibotti (2009). "Technological Change and the Wealth of Nations," Annual Review of Economics, 1, 93-120.

[33] Gilbert, Richard. and Carl Shapiro (1990), "Optimal Patent Length and Breadth," Journal of Industrial Economics, 21(1), 106-112.

[34] Greenwood, Jeremy and Mehmet Yorukoglu (1997), "1974," Carnegie-Rochester Conference Series on Public Policy, 46, 49-95.

[35] Grossman, Gene and Elhanan Helpman (1991). Innovation and Growth in the World Economy, MIT Press, Cambridge.

[36] Grossman, Gene and Edwin Lai (2004). "International Protection of Intellectual Property," American Economic Review 94, 1635-1653.

[37] Helpman, Elhanan (1993), "Innovation, Imitation and Intellectual Property Rights," Econometrica 61, 1247-1280.

[38] Howitt, Peter (2000). "Endogenous Growth and Cross-Country Income Differences" American Economic Review, 90, 829-846.

[39] Jovanovic, Boyan (2009). "The Technology Cycle and Inequality," Review of Economic Studies, 76, p 707-729. 
[40] Jovanovic, Boyan and Glenn MacDonald (1994). "The Life Cycle of a Competitive Industry," Journal of Political Economy 102, 322-347.

[41] Jovanovic, Boyan and Saul Lach (1989) "Entry, Exit, and Diffusion with Learning by Doing," American Economic Review, 79, 690-699.

[42] Jovanovic, Boyan and Yaw Nyarko (1996) "Learning by Doing and the Choice of Technology," Econometrica, 64, 1299-1310.

[43] Klemperer, Paul (1990), "How Broad Should the Scope of Patent Protection Be?" Journal of Industrial Economics, 21, 113-130.

[44] Krugman, Paul (1979) "A Model of Innovation, Technology Transfer, and the World Distribution of Income," Journal of Political Economy, 87, 253-266.

[45] Krusell, Per, Lee Ohanian, Victor Rios-Rull and Gianluca Violante (2000), "Capital Skill Complementarity and Inequality," Econometrica, 1029-1053.

[46] Lai, Edwin (1998). "International Intellectual Property Rights Protection and the Rate of Product Innovation," Journal of Development Economics 55, 115-130.

[47] Levin, Richard, Alvin Klevorick, Richard Nelson and Sidney Winter (1987). "Appropriating the Returns from Industrial Research and Development," Brookings Papers on Economic Activity, 3, 783-831.

[48] Loury, Glenn (1979). "Market Structure and Innovation," Quarterly Journal of Economics 93, 395-410.

[49] Nelson Richard, Edmund Phelps (1966). "Investment in humans, technological diffusion, and economic growth," American Economic Review 56, 69-75.

[50] Parente, Stephen and Edward Prescott (1994) "Barriers to Technology Adoption and Development" Journal of Political Economy, 102, 298-321.

[51] Romer, Paul (1990), "Endogenous Technological Change," Journal of Political Economy 98, 71-102.

[52] Segerstrom, Paul, T.C.A. Anant and Elias Dinopoulos (1990), "A Schumpeterian Model of the Product Life Cycle," American Economic Review 80, 1077-1091.

[53] Scotchmer, Suzanne (1999), "On the Optimality of the Patent System," Rand Journal of Economics, 30, 181-196.

[54] Stokey, Nancy (1991), "Human Capital, Product Quality, and Growth," Quarterly Journal of Economics 106, 587-616. 
[55] Utterback, James, and Fernando Suárez (1993), "Innovation, Competition, and Industry Structure," Research Policy 22, 1-21.

[56] Vernon, R. (1966). "International Investment and International Trade in ProductCycle," Quarterly Journal of Economics 80, 190-207.

[57] Yang, Guifang and Keith E. Maskus (2001). "Intellectual property rights, licensing and innovation in an endogenous product-cycle model" Journal of International Economics 53, 169-187. 


\section{Online Appendix For "Competing Engines of Growth: Innovation and STANDARDIZATION"}

Proof of Lemma 5

We take a linear approximation of the dynamical system $(40)$ around $\left(n^{s s}, \chi^{s s}\right)$ :

$$
\begin{aligned}
& \frac{\dot{\chi}}{\chi} \simeq F_{\chi}\left(\chi^{s s}, n^{s s}\right) \cdot\left(\chi-\chi^{s s}\right)+F_{n}\left(\chi^{s s}, n^{s s}\right) \cdot\left(n-n^{s s}\right) \\
& \frac{\dot{n}}{n} \simeq G_{\chi}\left(\chi^{s s}, n^{s s}\right) \cdot\left(\chi-\chi^{s s}\right)+G_{n}\left(\chi^{s s}, n^{s s}\right) \cdot\left(n-n^{s s}\right)
\end{aligned}
$$

where subscripts denote partial derivatives and

$$
\begin{aligned}
F(\chi, n) & \equiv r(n)-\rho-\frac{y(n)-\mu_{L} m(n) n-\chi}{\mu_{H}} \\
G(\chi, n) & \equiv\left(\frac{1-n}{n}\right) \frac{y(n)-\chi}{\mu_{H}}-m(n)\left(1+(1-n) \frac{\mu_{L}}{\mu_{H}}\right) .
\end{aligned}
$$

Thus, $F_{\chi}(\chi, n)=\frac{1}{\mu_{H}}>0$ and $G_{\chi}(\chi, n)=-\left(\frac{1-n^{s s}}{n^{s s}}\right) \frac{1}{\mu_{H}}<0$. Solving for the schedules such that, respectively, $\dot{\chi}=0$ and $\dot{n}=0$ yields:

$$
\begin{aligned}
\left.\chi(n)\right|_{\dot{\chi}=0} & =y(n)-\mu_{L} m(n) n-\mu_{H}(r(n)-\rho) \\
\left.\chi(n)\right|_{\dot{n}=0} & =y(n)-\mu_{L} m(n) n-\mu_{H} m(n)\left(\frac{n}{1-n}\right),
\end{aligned}
$$

with slopes:

$$
\left.\chi^{\prime}(n)\right|_{\dot{\chi}=0}=-\frac{F_{n}\left(\chi^{s s}, n^{s s}\right)}{F_{\chi}\left(\chi^{s s}, n^{s s}\right)} \quad \text { and }\left.\quad \chi^{\prime}(n)\right|_{\dot{n}=0}=-\frac{G_{n}\left(\chi^{s s}, n^{s s}\right)}{G_{\chi}\left(\chi^{s s}, n^{s s}\right)} .
$$

Suppose there were trajectories featuring both innovation and standardization converging to $\left(n^{s s}, \chi^{s s}\right)$. Then, either one or both eigenvalues of the linearized system would be negative. We show that this is impossible and that under the sufficient conditions of the Proposition both eigenvalues must be positive. Let the two eigenvalues of the linearized system be denoted by $\lambda_{1}$ and $\lambda_{2}$. We know that

$$
\begin{aligned}
\lambda_{1}+\lambda_{2} & =F_{\chi}\left(\chi^{s s}, n^{s s}\right)+G_{n}\left(\chi^{s s}, n^{s s}\right) \\
\lambda_{1} \cdot \lambda_{2} & =F_{\chi}\left(\chi^{s s}, n^{s s}\right) \cdot G_{n}\left(\chi^{s s}, n^{s s}\right)-F_{n}\left(\chi^{s s}, n^{s s}\right) \cdot G_{\chi}\left(\chi^{s s}, n^{s s}\right) .
\end{aligned}
$$

Claim 1 The following inequality holds

$$
-\frac{F_{n}\left(\chi^{s s}, n^{s s}\right)}{F_{\chi}\left(\chi^{s s}, n^{s s}\right)}<-\frac{G_{n}\left(\chi^{s s}, n^{s s}\right)}{G_{\chi}\left(\chi^{s s}, n^{s s}\right)} .
$$


Hence, $\lambda_{1} \cdot \lambda_{2}>0$

Proof. We need to show that $\left.\chi^{\prime}\left(n^{s s}\right)\right|_{\dot{\chi}=0}<\left.\chi^{\prime}\left(n^{s s}\right)\right|_{\dot{n}=0}$. Define $\Delta(n)=\left.\chi(n)\right|_{\dot{\chi}=0}-$ $\left.\chi(n)\right|_{\dot{n}=0}=\mu_{H} m(n)\left(\frac{n}{1-n}\right)-\mu_{H}(r(n)-\rho)$. We know that $m(n)=0$ for $n \geq n^{\max }>$ $n^{s s}$. Thus, at $n=n^{\max }$ we have:

$$
\Delta\left(n^{\max }\right)=-\mu_{H}(r(n)-\rho)=-\mu_{H}\left(\frac{\pi_{H}(n)}{\mu_{H}}-\rho\right)<0
$$

by Assumption 1. Next, recall that at $n^{\min }=\frac{H}{H+L}$ we have $y(n)=H+L, m(n)=$ $\frac{H+L}{\epsilon}\left(\frac{1}{\mu_{H}}-\frac{1}{\mu_{L}}\right)$ and $r(n)=\frac{H+L}{\epsilon \mu_{L}}$. Thus:

$$
\Delta\left(n^{\min }\right)=\mu_{H} \frac{H+L}{\epsilon}\left(\frac{1}{\mu_{H}}-\frac{1}{\mu_{L}}\right) h-\mu_{H}\left(\frac{H+L}{\epsilon \mu_{L}}-\rho\right)>0
$$

by Assumption 2. Moreover, we know that $n^{s s}$ is unique. Thus, $\left.\chi\left(n^{s s}\right)\right|_{\dot{\chi}=0}=\left.\chi\left(n^{s s}\right)\right|_{\dot{n}=0}$ for a unique value of $n^{s s}$. Then, by the intermediate value theorem, $\left.\chi(n)\right|_{\dot{\chi}=0}>\left.\chi(n)\right|_{\dot{n}=0}$ for all $n<n^{s s}$ and $\left.\chi(n)\right|_{\dot{\chi}=0}<\left.\chi(n)\right|_{\dot{n}=0}$ for all $n>n^{s s}$.

Since we know that $\lambda_{1} \cdot \lambda_{2}>0$, showing that $\lambda_{1}+\lambda_{2}>0$ establishes that the system has two positive eigenvalues and is therefore unstable. The condition $\lambda_{1}+\lambda_{2}>0$ can be written as:

$$
\left.\chi^{\prime}(n)\right|_{\dot{n}=0}=-\frac{G_{n}\left(\chi^{s s}, n^{s s}\right)}{G_{\chi}\left(\chi^{s s}, n^{s s}\right)}>\frac{F_{\chi}\left(\chi^{s s}, n^{s s}\right)}{G_{\chi}\left(\chi^{s s}, n^{s s}\right)}=-\left(\frac{n}{1-n}\right) \frac{1}{\mu_{H}} .
$$

A sufficient condition for (43) to be satisfied is that the locus $\dot{n}=0$ be upward sloping in a neighborhood of the BGP. Let us first consider the case where $\rho \rightarrow 0$. Using $y(n)=\epsilon\left[n \pi_{H}(n)+(1-n) \pi_{L}(n)\right]$ and $m(n)=\frac{\pi_{H}(n)}{\mu_{H}}-\frac{\pi_{L}(n)}{\mu_{L}}$ into $\chi(n)_{\mid \dot{n}=0}$ :

$$
\left.\chi(n)\right|_{\dot{n}=0}=y(n)-m(n)\left(\frac{n}{1-n} \mu_{H}+n \mu_{L}\right)=\left[\pi_{H}(n) n\right] \cdot A(n)
$$

where $A(n) \equiv\left[\frac{\mu_{L}}{\mu_{H}}+\frac{\pi_{L}(n)}{\pi_{H}(n)}\left(1+\frac{\epsilon}{n}\right)+\frac{1}{1-n}\left(\frac{\pi_{L}(n)}{\pi_{H}(n)} \frac{\mu_{H}}{\mu_{L}}-1\right)\right]$. We know that the factor $\pi_{H}(n) n$ is inverted U-shaped, with a maximum at $n^{*}>n_{\min }$ (it corresponds to the maximum $g$ characterized in Section 2.7). Thus, for $n \in\left[n_{\min }, n^{*}\right]$ a sufficient condition for $\chi^{\prime}(n)_{\mid \dot{n}=0}>0$ is $\frac{\partial A(n)}{\partial n}>0$ :

$\frac{\partial A(n)}{\partial n}=\frac{\partial}{\partial n}\left(\frac{\pi_{L}(n)}{\pi_{H}(n)}\right)\left(1+\frac{\epsilon}{n}\right)-\frac{\pi_{L}(n)}{\pi_{H}(n)} \frac{\epsilon}{n^{2}}+\frac{1}{(1-n)^{2}}\left(\frac{\pi_{L}(n)}{\pi_{H}(n)} \frac{\mu_{H}}{\mu_{L}}-1\right)+\frac{\partial}{\partial n}\left(\frac{\pi_{L}(n)}{\pi_{H}(n)}\right) \frac{1}{1-n}$ 
For $\rho \rightarrow 0$, in the BGP we have $\frac{\pi_{L}(n)}{\pi_{H}(n)}=\frac{\mu_{L}}{\mu_{H}} n$ and $\frac{\partial}{\partial n}\left(\frac{\pi_{L}(n)}{\pi_{H}(n)}\right)=\frac{\epsilon-1}{\epsilon} \frac{\pi_{L}(n)}{\pi_{H}(n)} \frac{1}{(1-n) n}=$ $\frac{\epsilon-1}{\epsilon(1-n)} \frac{\mu_{L}}{\mu_{H}}$. Thus:

$$
\frac{\partial A(n)}{\partial n}=\frac{\mu_{L}}{(1-n) \mu_{H}}\left[\frac{\epsilon-1}{\epsilon}+\epsilon-\frac{1}{n}+\frac{\epsilon-1}{\epsilon(1-n)}+\frac{\mu_{H}}{\mu_{L}}\right] .
$$

A sufficient condition for $\left.\chi^{\prime}(n)\right|_{\dot{n}=0}>0$ is then: $\frac{\epsilon-1}{\epsilon}+\epsilon-\frac{1}{n}+\frac{\epsilon-1}{\epsilon(1-n)}+\frac{\mu_{H}}{\mu_{L}}>0$. Noting that in the BGP $\frac{\pi_{L}(n)}{\pi_{H}(n)}=\frac{\mu_{L}}{\mu_{H}} n \rightarrow \frac{\mu_{H}}{\mu_{L}}=\left(\frac{1-n}{n} h\right)^{\frac{\epsilon-1}{\epsilon}} n$, the sufficient condition become:

$$
\frac{\epsilon-1}{\epsilon}+\epsilon-\frac{1}{n}+\frac{\epsilon-1}{\epsilon(1-n)}+\left(\frac{1-n}{n} h\right)^{\frac{\epsilon-1}{\epsilon}} n>0
$$

Since this expression is increasing in $n$, we only need to verify that it is positive at $n_{\min }$. At $n_{\min }$ we have $\left(\frac{1-n}{n} h\right)^{\frac{\epsilon-1}{\epsilon}}=1$ and the condition becomes:

$$
\frac{\epsilon-1}{\epsilon}+\epsilon-\frac{1}{n}+\frac{\epsilon-1}{\epsilon(1-n)}+n>0
$$

Substituting $n_{\min }=h /(1+h)$ yields $(28)$ in the text.

Finally, for $n>n^{*}$, rewrite the necessary condition as:

$$
\frac{\left.\chi^{\prime}(n)\right|_{\dot{n}=0}}{\left.\chi(n)\right|_{\dot{n}=0}}=\frac{\pi_{H}^{\prime}(n)}{\pi_{H}(n)}+\frac{1}{n}+\frac{\partial A}{\partial n} \frac{1}{A}>-\left(\frac{n}{1-n}\right) \frac{1}{\left.\mu_{H} \chi(n)\right|_{\dot{n}=0}}
$$

From equations (16) and (17):

$$
\frac{\pi_{H}^{\prime}(n)}{\pi_{H}(n)}+\frac{1}{n}=\frac{1}{\epsilon} \frac{[y(n)]^{\frac{1-\epsilon}{\epsilon}}}{\epsilon-1}\left(\frac{H}{n}\right)^{\frac{\epsilon-1}{\epsilon}}-\frac{1}{\epsilon} \frac{[y(n)]^{\frac{1-\epsilon}{\epsilon}}}{\epsilon-1}\left(\frac{L}{1-n}\right)^{\frac{\epsilon-1}{\epsilon}}+\frac{1}{n \epsilon} .
$$

Substituting $[y(n)]^{\frac{\epsilon-1}{\epsilon}}=(1-n)^{\frac{1}{\epsilon}} L^{\frac{\epsilon-1}{\epsilon}}+n^{\frac{1}{\epsilon}} H^{\frac{\epsilon-1}{\epsilon}}$ into this expression, we have

$$
-\frac{1}{\epsilon(\epsilon-1)} \frac{(1-n)^{-1}}{1+\left(\frac{n}{1-n}\right)^{\frac{1}{\epsilon}} h^{\frac{\epsilon-1}{\epsilon}}}<\frac{\pi_{H}^{\prime}(n)}{\pi_{H}(n)}+\frac{1}{n}
$$

This implies that $\frac{\partial A(n)}{\partial n} \frac{1}{A(n)}>\frac{1}{\epsilon(\epsilon-1)} \frac{\frac{1}{1-n}}{1+\left(\frac{n}{1-n}\right)^{\frac{1}{\epsilon}} h^{\frac{\epsilon-1}{\epsilon}}}$ is a sufficient condition for $\left.\chi^{\prime}(n)\right|_{\dot{n}=0}>$ 0 . Using (44) and the fact that in BGP $\frac{\pi_{L}(n)}{\pi_{H}(n)}=\frac{\mu_{L}}{\mu_{H}} n$, it can be verified that this condition is satisfied when (45) holds, for any $n \in\left[n^{*}, 1\right]$. In sum, (45) is sufficient to prove that the dynamical system with both innovation and standardization is locally unstable in the limit where $\rho \rightarrow 0$. By continuity, the same result applies for $\rho<\bar{\rho}$ for some $\bar{\rho}>0$ sufficiently small. 